\title{
Impaired angiopoietin/Tie2 signaling compromises Schlemm's canal integrity and induces glaucoma
}

\author{
Jaeryung Kim,, ${ }^{1}$ Dae-Young Park, ${ }^{1,2,3}$ Hosung Bae, ${ }^{1}$ Do Young Park, ${ }^{1}$ Dongkyu Kim, ${ }^{2}$ Choong-kun Lee, ${ }^{1}$ Sukhyun Song, ${ }^{2}$ \\ Tae-Young Chung, ${ }^{3}$ Dong Hui Lim, ${ }^{3,4}$ Yoshiaki Kubota, ${ }^{5}$ Young-Kwon Hong, ${ }^{6}$ Yulong He, ${ }^{7}$ Hellmut G. Augustin, ${ }^{8}$ Guillermo Oliver, ${ }^{9}$ \\ and Gou Young Koh ${ }^{1,2}$ \\ 'Craduate School of Medical Science and Engineering, Korea Advanced Institute of Science and Technology (KAIST), Daejeon, Republic of Korea. '2Center for Vascular Research, Institute for Basic Science, \\ Daejeon, Republic of Korea. ${ }^{3}$ Department of Ophthalmology, Samsung Medical Center, Sungkyunkwan University School of Medicine, Seoul, Republic of Korea. ${ }^{4}$ Department of Preventive Medicine, \\ Catholic University School of Medicine, Seoul, Republic of Korea. ${ }^{5}$ The Laboratory of Vascular Biology, School of Medicine, Keio University, Tokyo, Japan. ${ }^{6}$ Department of Surgery, Department of Biochemistry \\ and Molecular Biology, Norris Comprehensive Cancer Center, Keck School of Medicine, University of Southern California, Los Angeles, California, USA. `Cyrus Tang Hematology Center, Collaborative Innovation \\ Center of Hematology, Soochow University, Suzhou, China. ${ }^{8}$ Division of Vascular Oncology and Metastasis, German Cancer Research Center Heidelberg (DKFZ-ZMBH Alliance), Heidelberg, Germany. \\ ${ }^{9}$ Center for Vascular and Developmental Biology, Northwestern University Feinberg School of Medicine, Chicago, Illinois, USA.
}

Primary open-angle glaucoma (POAC) is often caused by elevated intraocular pressure (IOP), which arises due to increased resistance to aqueous humor outflow (AHO). Aqueous humor flows through Schlemm's canal (SC), a lymphatic-like vessel encircling the cornea, and via intercellular spaces of ciliary muscle cells. However, the mechanisms underlying increased AHO resistance are poorly understood. Here, we demonstrate that signaling between angiopoietin (Angpt) and the Angpt receptor Tie2, which is critical for SC formation, is also indispensable for maintaining SC integrity during adulthood. Deletion of Angpt1/Angpt2 or Tie2 in adult mice severely impaired SC integrity and transcytosis, leading to elevated IOP, retinal neuron damage, and impairment of retinal ganglion cell function, all hallmarks of POAC in humans. We found that SC integrity is maintained by interconnected and coordinated functions of Angpt-Tie2 signaling, AHO, and Prox1 activity. These functions diminish in the SC during aging, leading to impaired integrity and transcytosis. Intriguingly, Tie2 reactivation using a Tie2 agonistic antibody rescued the POAG phenotype in Angpt1/Angpt2-deficient mice and rejuvenated the SC in aged mice. These results indicate that the Angpt-Tie2 system is essential for SC integrity. The impairment of this system underlies POAGassociated pathogenesis, supporting the possibility that Tie2 agonists could be a therapeutic option for glaucoma.

\section{Introduction}

Glaucoma is a leading cause of irreversible blindness, affecting $3.5 \%$ of the population aged 40 to 80 years worldwide (1). It involves cupping of the optic nerve head and irreversible loss of retinal ganglion cells, mainly because of chronic, elevated intraocular pressure (IOP) caused by impairment of aqueous humor outflow (AHO) (2). Aqueous humor (AH) is constantly produced by the ciliary body in the posterior chamber, flows into the anterior chamber, and exits the eye either through the trabecular meshwork (TM) into the Schlemm's canal (SC) and aqueous and episcleral veins (conventional AHO pathway [AHOc]; $\geq 75 \%$ ) or through the intercellular spaces of the ciliary muscle cells (unconventional AHO pathway $[\mathrm{AHOu}] ;<25 \%)(3,4)$. The direction of the $\mathrm{AH}$ in $\mathrm{AHOc}$ is basal to apical, like that of the interstitial fluid into the lymphatic capillary ends at peripheral tissues. In acquired adult-onset primary open-angle glaucoma (POAG), the most common form of

\section{Related Commentary: p. 3594}

Authorship note: J. Kim and D.Y. Park contributed equally to this work Conflict of interest: The authors have declared that no conflict of interest exists. Submitted: April 21, 2017; Accepted: July 18, 2017.

Reference information: J Clin Invest. 2017;127(10):3877-3896.

https://doi.org/10.1172/JCI94668. glaucoma, constituting up to three-quarters of all diagnosed cases (1), an abnormally high resistance against AHOc is responsible for increased IOP and subsequent optic neuropathy (5). Nevertheless, current conventional treatments for glaucoma that act by controlling production or outflow of $\mathrm{AH}$ have shown poor outcomes thus far. Moreover, the precise molecular mechanism underlying the increased outflow resistance is poorly understood.

SC is an encircling channel consisting of continuous, nonfenestrated endothelial cell (EC) monolayers at the periphery of the cornea $(4,6)$ and shares morphological, functional, and molecular similarities with lymphatic vessels (LVs) (7-10). Similar to the thoracic duct, which drains lymph into the subclavian vein through the lymphovenous valve (11), the SC constitutes a blind-ended tube that transfers $\mathrm{AH}$ and antigen-presenting cells to the systemic circulation through the episcleral vein $(12,13)$. Moreover, connecting fibrils stretching from the SC ECs into the surrounding cribriform plexus play a role in preventing SC collapse (14), similar to that of the LVanchoring filaments $(6,15)$. Recently, we and others found that the SC identity is a mixture between LV and blood vessel (BV), as indicated by the expression of the lymphatic markers Prox1, VEGFR3, and integrin $\alpha 9$ (no LYVE-1 and podoplanin) and blood markers such as Tie2 (7-10). The SC originates from the choroidal vein, and postnatally, SC ECs acquire a lymphatic fate by the upregulation of Prox1 (8-10), a master transcriptional factor of lymphatic ECs (LECs) (16, 
A
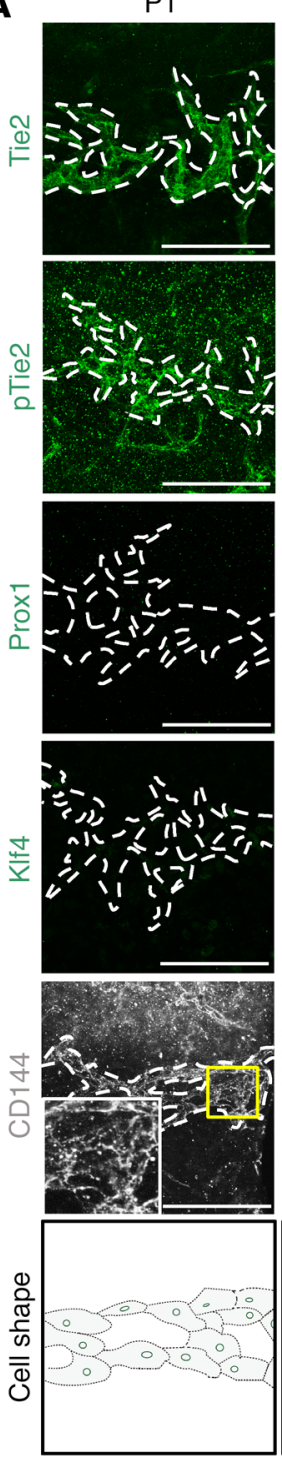

P4
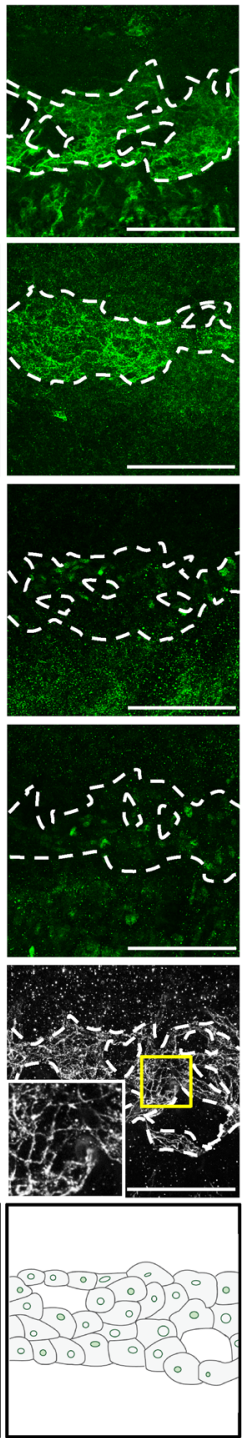

P7
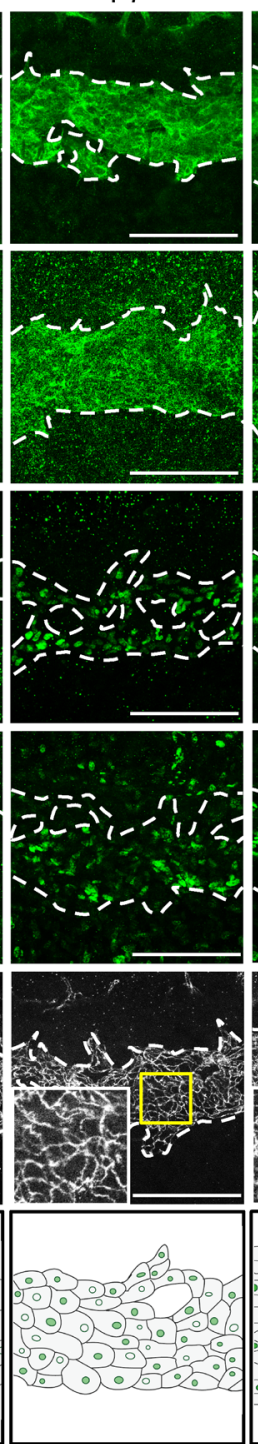

P14
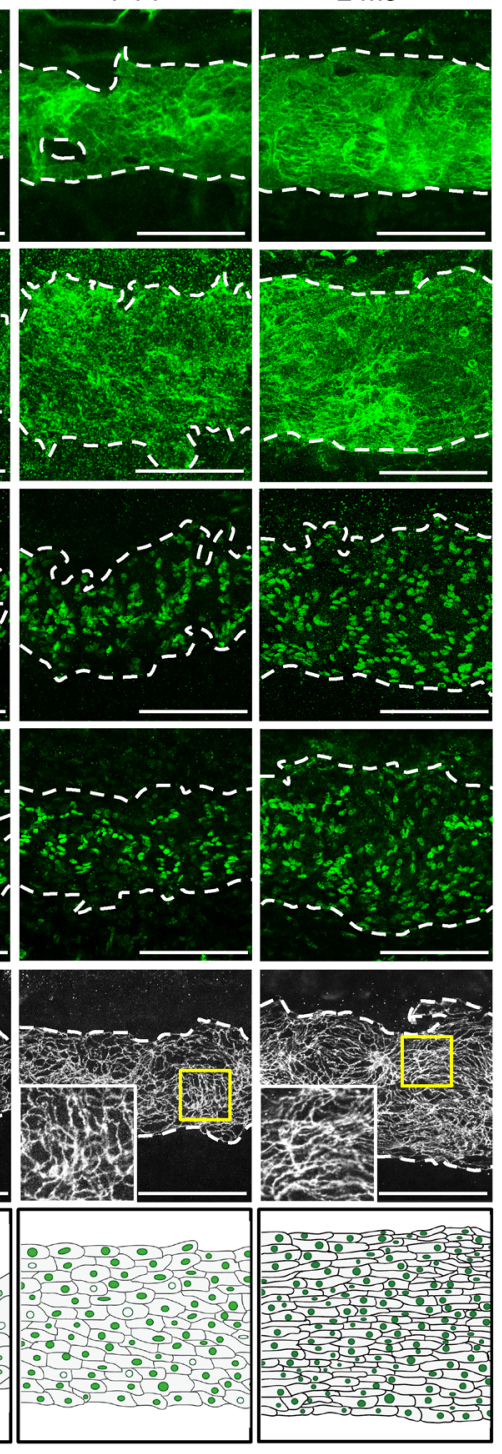

$2 \mathrm{mo}$
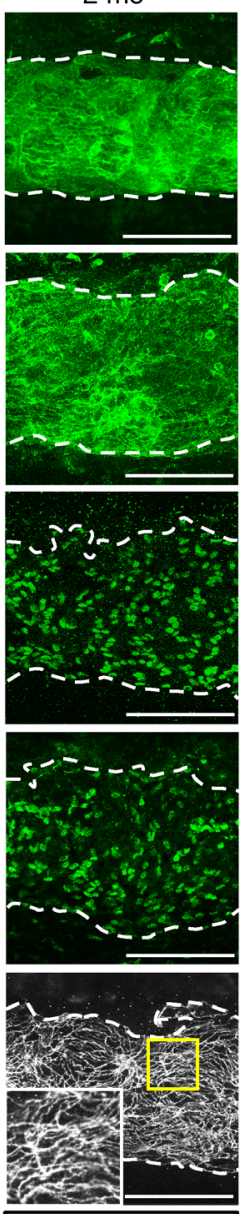

B $\mathrm{PO}$

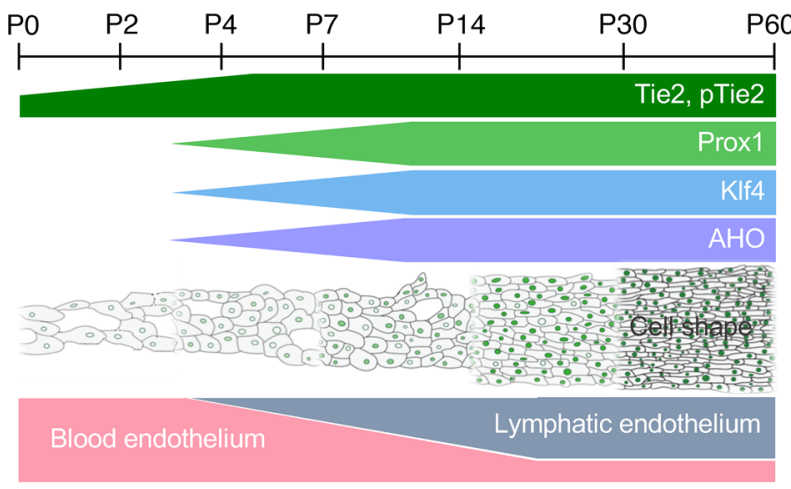

17). The expression of Prox 1 as a molecular biosensor reflects the integrity and functionality of the SC (8); however, the role of Prox1 in the formation and maintenance of the SC is unknown.

The angiopoietin-Tie2 (Angpt-Tie2) pathway controls vascular maturation and stabilization (18-20). Angpt1 acts as an agonist to Tie2, while Angpt2 acts as an agonist and an antagonist to Tie2 in a context-dependent manner (18). Prenatal deletion of Angpt1 in mice leads to impairment in cardiovascular development (21), and prenatal deletion or inhibition of Angpt2 in mice causes defects in lymphatic maturation (22-24). Prenatal deletion of Tie2 also leads to severe impairments in cardiovascular development, including vein specification and venogenesis $(18,25)$. Regarding the SC, Quaggin and colleagues elegantly showed that the Angpt-Tie2 system is involved in SC formation, as proven with postnatal double Angpt1/Angpt2-deleted or Tie2-deleted mice that exhibited primary congenital glaucoma (26). Moreover, Tie2 mutations have been 
A $\quad$ CD31 TIE2

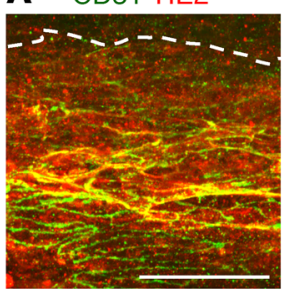

PROX1 CD144

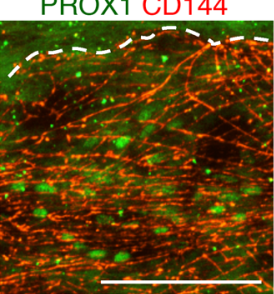

B Tie2-GFP PDGFR $\beta$

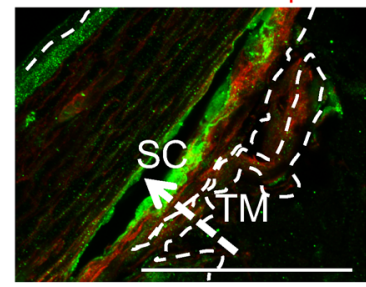

Prox1-GFP Col IV

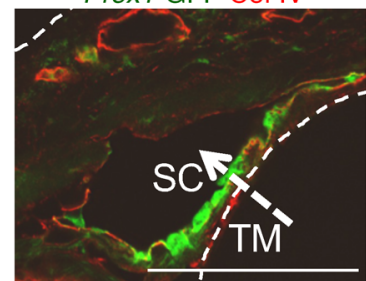

C

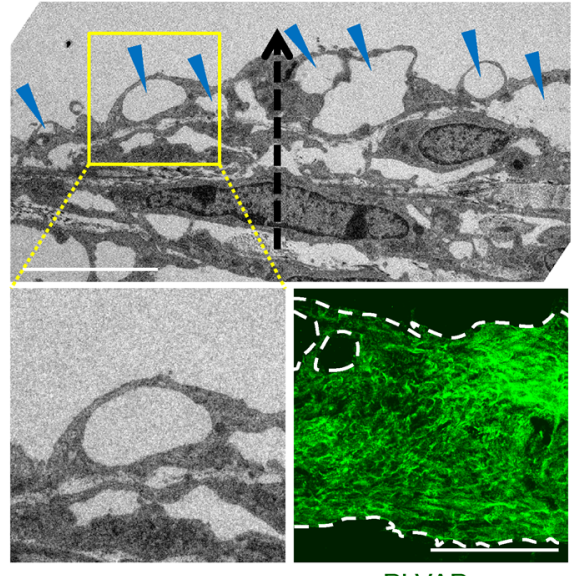

PLVAP
D

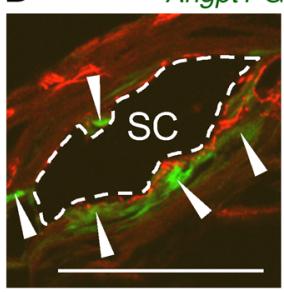

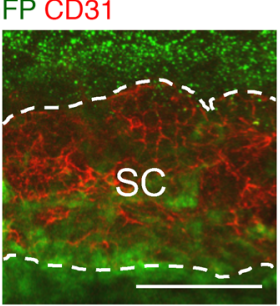

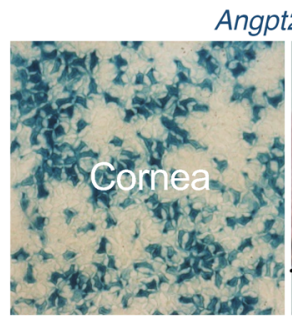

Angpt2-lacZ

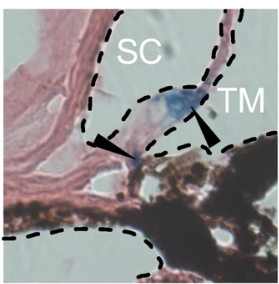

E

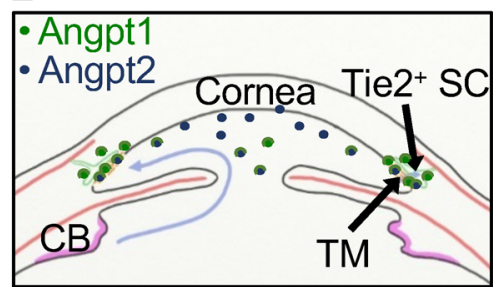

Figure 2. Tie2 in the inner SC wall is constantly exposed to Angpts. (A) Images showing PROX1 and TIE2 in healthy adult human SCs. Scale bars: 100 um. (B) Cross-section images showing Prox $1^{+}$and Tie2 ${ }^{+}$SC ECs and distribution of collagen IV or PDCFR $\beta$ in corneal limbus of 2-month-old Prox1-GFP and Tie2GFP mice. Dashed arrows indicate direction of AHO. Scale bars: $100 \mu \mathrm{m}$. (C) EM images of SC and distribution of PLVAP in the ECs of the inner SC walls in 2-month-old mice. Yellow-lined box is magnified in left lower panel. Blue arrowheads indicate GVs in the inner SC wall, and dashed arrow indicates the direction of AHO. Scale bars: $5 \mu \mathrm{m}$ (upper panel); $100 \mu \mathrm{m}$ (right lower panel). (D) Images showing expression of Angpt1 (white arrowheads) in pericytes of SC and Angpt2 in corneal endothelium and TM (black arrowheads) in 2-month-old Angpt1-GFP and Angpt2-lacZ mice. Scale bars: 100 $\mu$ m. (E) Diagram depicting the sources and distributions of secreted Angpt1 and Angpt2 for the formation and maintenance of Tie2+ SC. CB, ciliary body.

identified in patients with primary congenital glaucoma (27). Considering these exciting findings, we decided to evaluate whether the Angpt-Tie2 system is also critically required for maintenance of SC integrity during adulthood and, if so, what the underlying mechanism is. Here, we show that Angpt-Tie2 signaling is indispensably required for maintaining adult SC integrity by maintaining normal AHO and Prox1 expression, as inactivation of this pathway during adulthood causes POAG-like phenotypes. Finally, Tie2 reactivation rescues the phenotypes of POAG in double Angpt1/ Angpt2-deleted mice and rejuvenates the SC in aged mice.

\section{Results}

Tie2 expression precedes Prox 1 expression in the SC. To gain insight about the role of Tie 2 signaling in SC formation, we first examined its normal expression in WT C57BL/6J mice. Tie2 and phosphorylated Tie2 at Tyr992 (hereafter named p-Tie2) were expressed in SC progenitors originating from the choroidal veins at $\mathrm{P} 1$, and their expression increased further during SC development (Figure 1, A and B). Expression of Prox1 and Klf4, key shear stress-responsive transcription factors $(28,29)$, became obvious at P7 and continued increasing, and VE-cadherin ${ }^{+} \mathrm{EC}$ junctions started to become distinct (Figure 1, A and B). As the SC differentiated, EC shapes converted from cuboidal to longitudinal (Figure 1, A and B), changes that appeared to correlate with gain of predicted AHO and lym- phatic phenotypes (8). Thus, Tie2 expression started prior to that of Prox 1 in the ECs of the SC before an acquisition of AHO.

Consistently, Tie2 and Prox1 were also highly detected in ECs of adult human SCs (Figure 2A). Cross-section images of the SC in Tie2-GFP and Prox1-GFP mice (30) revealed that Tie2 and Prox1 expression were higher in the ECs located in the inner SC wall (Figure 2B), the principal route of AHO (31). Variable-sized multiple giant vacuoles (GVs), which are outpouchings of SC ECs that bulge into the SC lumen and proportionally reflect AHOc levels (32), and abundant plasmalemma vesicle-associated protein (PLVAP), a marker for endothelial transcytosis and permeability, were detected in the ECs of the inner SC wall (Figure 2C), reflecting active transcellular transport of $\mathrm{AH}$ through the SC. Analyses using Angpt1-GFP mice (33) and Angpt2-lacZ mice (22) showed that Angpt1 was expressed in pericytes of the SC, whereas Angpt2 was expressed in the trabecular region and corneal endothelium (Figure 2D). This result implies that Tie2 in the SC is constantly exposed to Angpt1 and Angpt2 secreted from the adjacent cells directly or via $\mathrm{AH}$ (Figure 2E).

Tie2 is required for SC development and maintenance. To determine the role of Tie2 in SC development, we generated a Tie $2^{i \Delta E C}$ mouse by crossing the VE-cadherin-Cre-ER ${ }^{\mathrm{T} 2}$ mouse (34) with the Tie $2^{f / f l}$ mouse (35). We then administered tamoxifen starting at $\mathrm{P} 1$ and analyzed the animals 2 months later (Figure 3A). 
A Tie $2^{\triangle \triangle E C}$ mouse

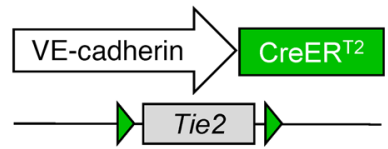

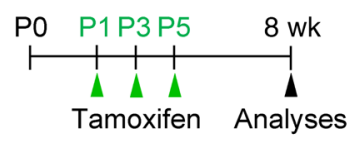
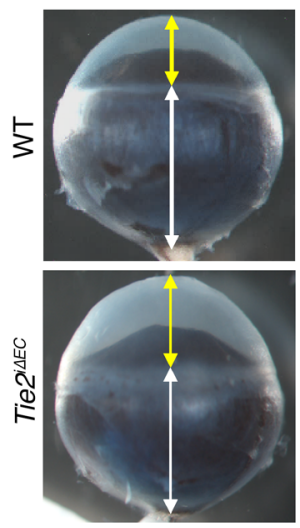

Tie2 CD144
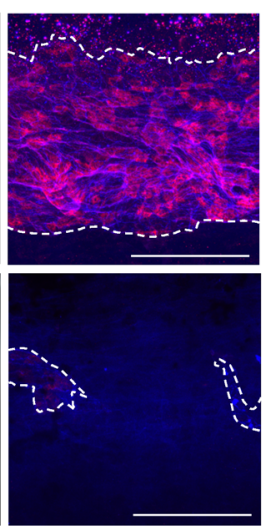

B

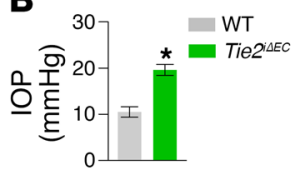

D

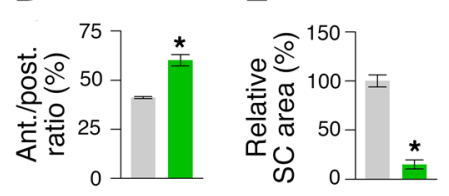

F

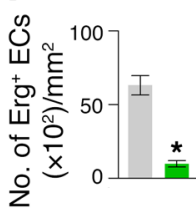

CD144

Erg
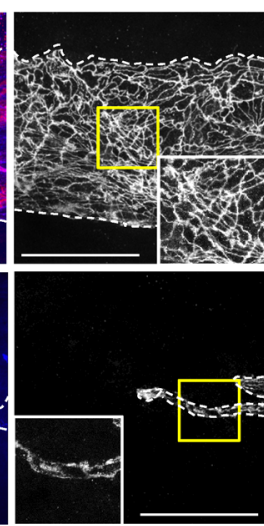
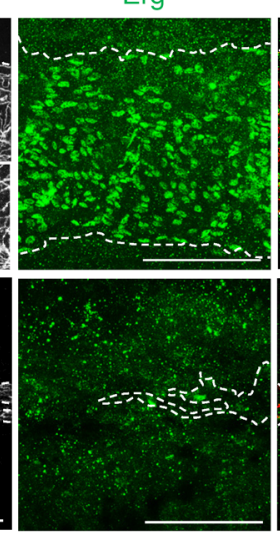

Prox1 CD144
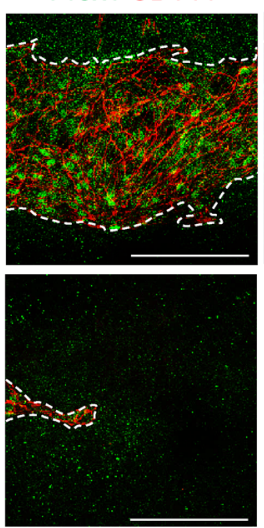

Klf4 CD31
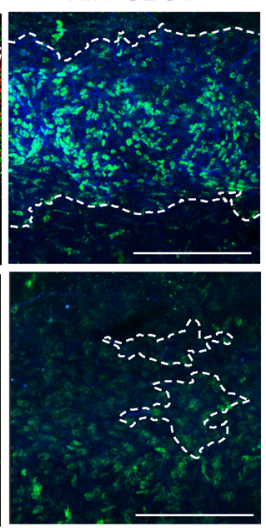

G

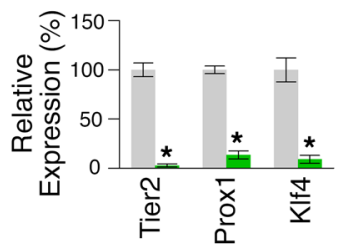

Figure 3. Tie2 is critical for SC generation. (A) Diagram for EC-specific depletion of Tie2 in SC starting at P1 and analyses 8 weeks later using Tie2 ${ }^{i 4 E C}$ mice. (B-C) Images and comparisons of IOP, an anterior (yellow double arrow)/posterior (white double arrow) (ant./post.) segment ratio of the eyeball, relative area, number of Erg' ECs, and intensities of Tie2, Prox1, and KIf4 immunostaining in CD144+ SC. Dashed lines demarcate the margins of SC. Each yellow-lined image is magnified in the corner. Scale bars: $100 \mu \mathrm{m}$. SC area and expression of each molecule in WT mice are normalized to $100 \%$, and their relative levels in Tiez ${ }^{\text {iAEC }}$ mice are presented. $n=4-5$ for each group. ${ }^{*} P<0.05$ versus WT by Mann-Whitney $U$ test.

Cre-ER ${ }^{\mathrm{T} 2}$-positive but flox/flox-negative mice among the littermates for each experiment were defined as WT mice. Elevated IOP with an elongated anterior-to-posterior segment ratio of the eyeball, resembling buphthalmos (eyes with an enlarged anterior segment) in humans, was detected in the eyes of Tie $2^{i \Delta E C}$ mice (Figure 3, B-D). Protein levels of Tie2, Prox1, and Klf4 and area and cellularity (number of $\mathrm{Erg}^{+} \mathrm{ECs}$ ) in SCs were markedly reduced in Tie $2^{\text {iSEC }}$ mice compared with WT mice (Figure 3, E-G). Electron microscopic (EM) analysis revealed that GVs were nearly absent in the inner SC walls of Tie ${ }^{i \Delta E C}$ mice (Figure 4, A and B), indicating a lack of $\mathrm{AHO}$ for maintaining a basal-to-apical gradient pressure in their SCs. Moreover, a thinned retinal nerve fiber layer (RNFL) and markedly attenuated positive scotopic threshold response (pSTR) and photopic negative response (PhNR) (measurements of these 2 responses are widely used to evaluate electrical function of retinal ganglion cells) were observed in Tie $2^{i \Delta E C}$ mice (Figure $4, \mathrm{C}-\mathrm{H}$ ), meaning that elevated IOP for a long term led to gradual neuronal damage in the retina and ultimately to visual impairment.

To address how Tie2 deletion impairs SC formation, we analyzed the SCs of Tie ${ }^{i \Delta E C}$ mice at P7 (Supplemental Figure 1A; supplemental material available online with this article; https:/doi. org/10.1172/JCI94668DS1). Compared with WT mice, Tie2 $2^{i \Delta E C}$ mice already had severely impaired SC formation together with marked reductions in limbal blood and lymphatic vascular densi- ties at the cornea (Supplemental Figure 1, B, C, and E-G). Moreover, Prox1 and Klf4 were markedly reduced in SCs, although their levels were similar in the limbal LVs of Tie ${ }^{i \Delta E C}$ mice compared with those of WT mice (Supplemental Figure 1, B and D), indicating that early Tie 2 activation is definitely required for SC formation.

To examine the role of Tie2 in maintenance of SC integrity, we deleted Tie2 in the SC ECs by administering tamoxifen into 8week-old Tie $2^{\text {iSEC }}$ mice and analyzing them 4 weeks later (Figure $5 \mathrm{~A}$ ). Unexpectedly, elevated IOP with a slightly but significantly elongated anterior-to-posterior segment ratio of the eyeball was detected in Tie $2^{i \Delta E C}$ mice (Figure 5, B-D). Protein levels of Tie2, Prox1, and Klf4 and area and cellularity in SCs were also reduced in Tie $2^{i \Delta E C}$ mice compared with those of WT mice (Figure 5, C and E-G). EM analysis revealed that the number and diameter of GVs were markedly decreased in SC ECs of Tie $2^{i \Delta E C}$ mice (Figure 6, A-C), indicating inadequate AHO in their SCs. Moreover, thinned RNFL and markedly attenuated pSTR and PhNR were observed in Tie $2^{\text {iSEC }}$ mice (Figure 6, D-I), implying that an elevated IOP for a month led to retinal neuronal damage and partial visual impairment. In contrast, no apparent changes were detected in the vessels of other organs in Tie $2^{\text {iSEC }}$ mice (Supplemental Figure 2, A-E). Moreover, when we deleted Vegfr2 in 8-week-old Vegfri ${ }^{\mathrm{LEC}}$ mice, the mice showed no apparent changes in IOP and SCs 4 weeks later (Supplemental Figure $3, \mathrm{~A}-\mathrm{F}$ ) in spite of the reduced vascularity and number of fenes- 
A

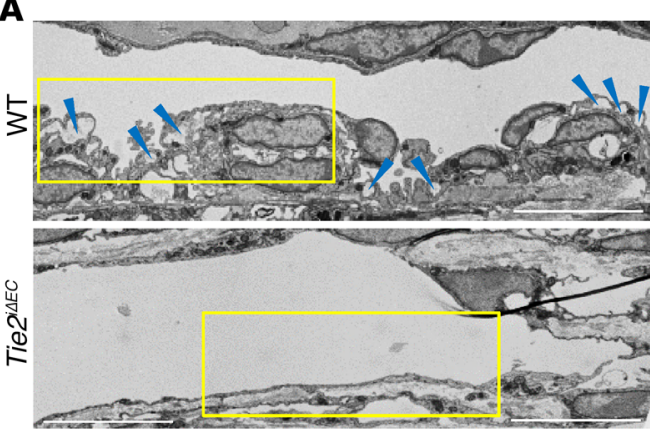

C

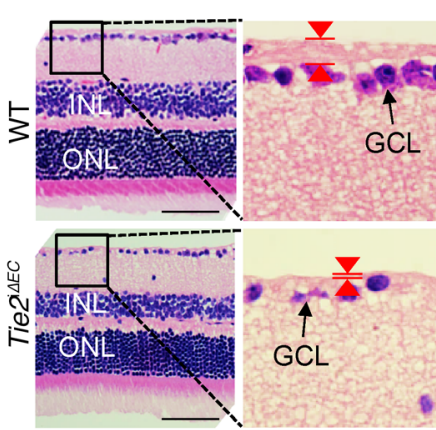

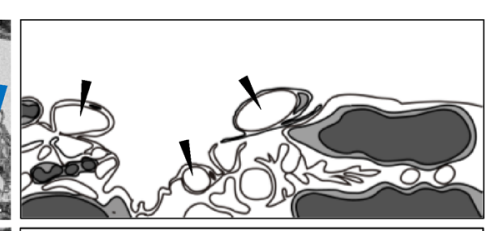

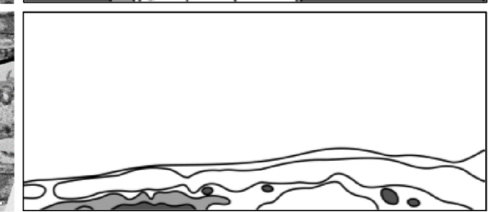

D
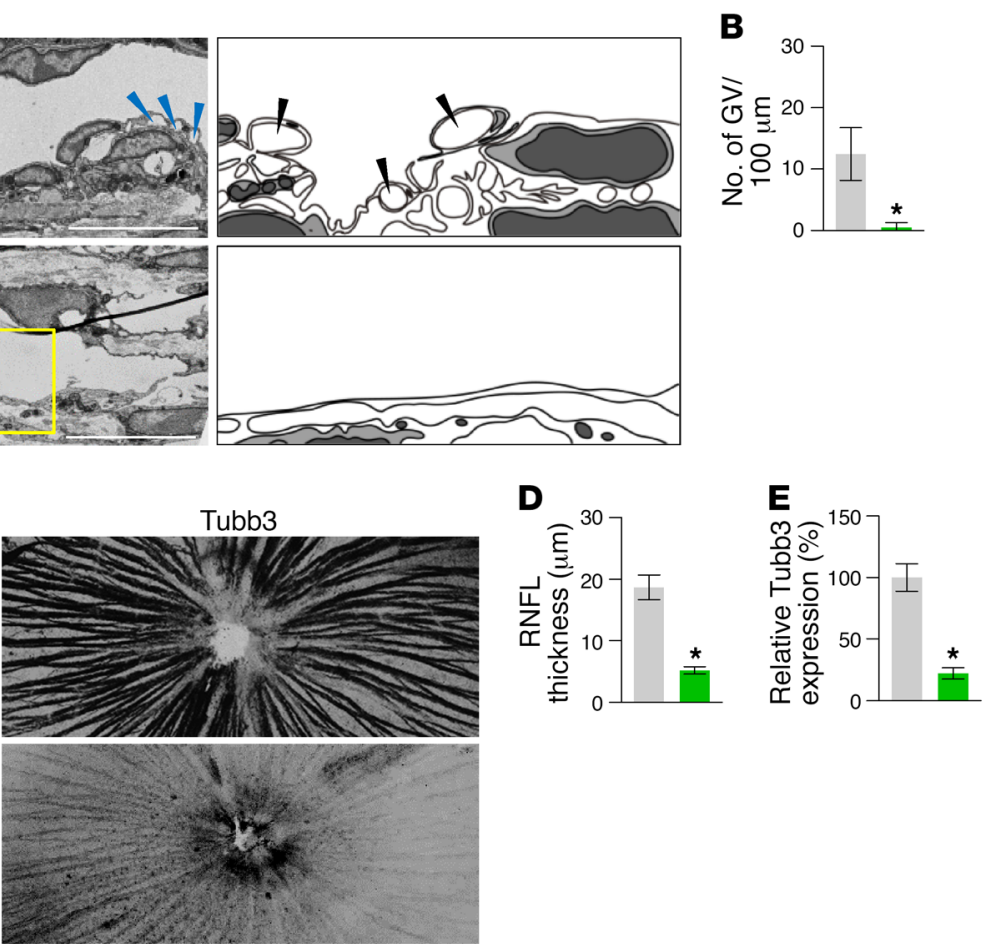

$\mathbf{F}$

Scotopic

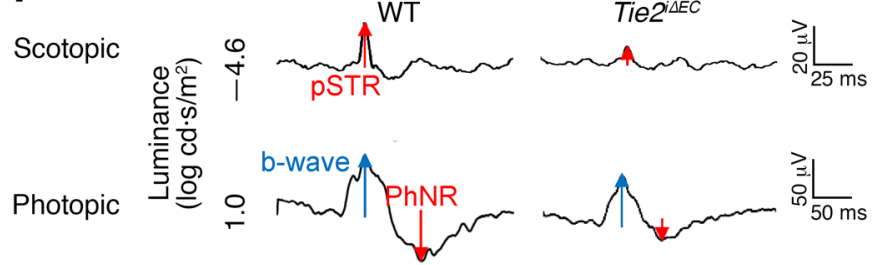

G

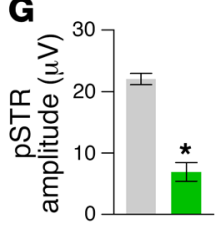

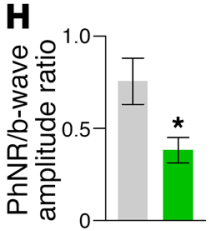

Figure 4. Inadequate SC development by Tie2 depletion impairs AHO and retinal ganglion cell function. (A and B) EM images and comparison of the number of GVs (blue and black arrowheads) in the inner SC wall. Right panels depict illustrative views of GVs. Scale bars: $10 \mu \mathrm{m}$. $n=4$ for each group. ${ }^{*} P<0.05$ versus WT by Mann-Whitney $U$ test. (C-E) Images and comparison of thickness (red arrowheads) and Tubb3 distribution of RNFL. Black-lined box is magnified in middle panel. INL, inner nuclear layer; ONL, outer nuclear layer; GCL, ganglion cell layer. Scale bars: $200 \mu \mathrm{m}$. (F-H) Representative wave responses of electroretinogram and comparisons of $\mathrm{pSTR}$ amplitude and PhNR/b-wave amplitude ratio. $n=4$ for each group. ${ }^{*} P<0.05$ versus WT by Mann-Whitney $U$ test.

trations in ECs of thyroid glands. Together, these findings suggest that Tie 2 is critical for maintenance of SC integrity during adulthood.

Angpt1 and Angpt2 play overlapping roles through Tie2 signaling in the generation and maintenance of the SC. To determine whether Angpt1 or Angpt2 could regulate development and maintenance of SC, we generated Angpt $1^{i \Delta / 4}$ and Angpt $2^{i / / 4}$ mice by crossing Angpt $1^{f / / l}(36,37)$ or Angpt $2^{f / f l}$ (38) mice with the ubiquitin-Cre-ER ${ }^{\mathrm{T} 2}$ mouse, depleted Angpt1 or Angpt 2 starting at P1 by tamoxifen administration, and analyzed the tamoxifen-treated mice at P14 (Supplemental Figure 4, A and G). Compared with WT mice, Angpt $1^{\mathrm{i} / / 4}$ mice exhibited markedly retarded SC growth, but slightly diminished limbal LVs (Supplemental Figure 4, C, D and F) and reduced Prox1, Vegfr3, and Klf4, but unchanged Tie2 in SC (Supplemental Figure 4, C and E). In comparison, Angpt $2^{i / / 4}$ mice showed a slightly diminished SC area, but severely retarded limbal LVs (Supplemental Figure 4, I, J, and L), and reduced Prox1, Vegfr3, and Klf4, but unchanged Tie2 in SC (Supplemental Figure 4 , I and K). Nevertheless, there were no changes in IOP in either mouse group (Supplemental Figure 4, B and H).
To assess the role of Angpt1 or Angpt2 in SC maintenance, we deleted each gene individually by tamoxifen administration into 8-week-old Angpt $1^{i / / 4}$ or Angpt $2^{i / / 4}$ mice and analyzed their SCs 4 weeks later (Supplemental Figure 5, A and G). No changes in IOP were found in either mouse strain (Supplemental Figure 5, B and $\mathrm{H})$. We detected mildly reduced cellularity and levels of Prox1 and Klf4, but not Tie2, in the SCs of both mouse strains, while SC area was slightly reduced only in Angpt $2^{i / /}$ mice (Supplemental Figure 5, C-F and I-L). These data imply that Angpt1 and Angpt2 play overlapping but somewhat different roles in the generation and maintenance of SC.

These findings led us to generate double Angpt1/Angpt2deficient $\left(A 1: A 2^{i / /}\right)$ mice. Compared with WT mice, SC formation was severely impaired together with markedly reduced Prox1, Klf4, Tie2, and p-Tie2 at P7 in the $A 1: A 2^{i / / 4}$ mice (Supplemental Figure 6, A-D). At 2 months of age, the $A 1: A 2^{i / / 4}$ mice had elevated IOP, with an elongated anterior-to-posterior segment ratio of the eyeball, markedly reduced area, cellularity, and protein levels of Prox1, Klf4, Tie2, and p-Tie2, and increased $\alpha \mathrm{SMA}^{+}$cells in 
A

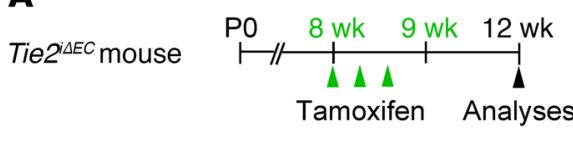

ill

C
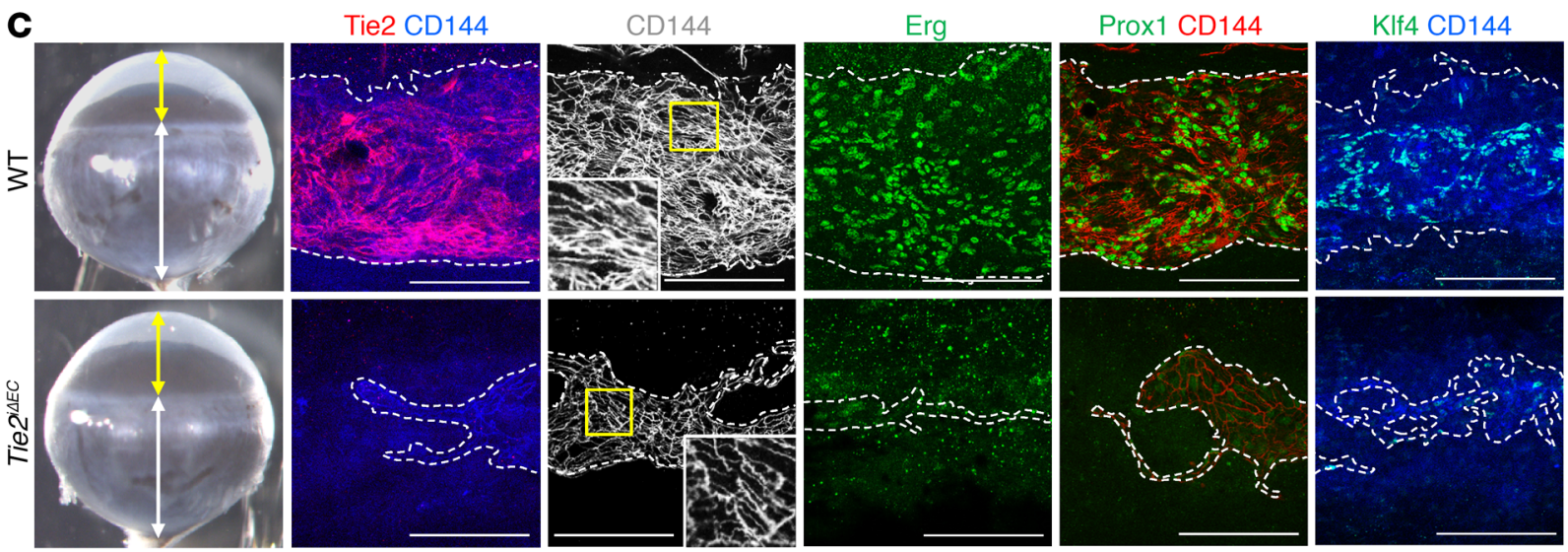
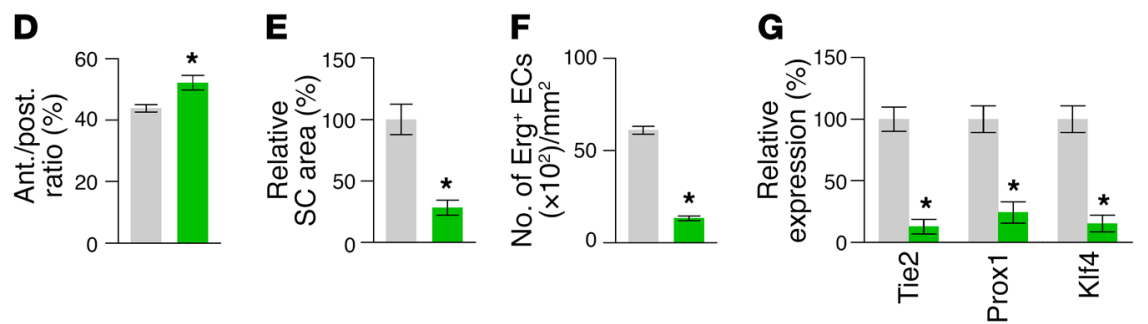

Figure 5. Tie2 is critical for maintenance of SC. (A) Diagram for EC-specific depletion of Tie2 in SC in 8-week-old mice and analyses 4 weeks later using Tie2 ${ }^{\text {ISEC }}$ mice. (B-G) Images and comparisons of IOP, an anterior (yellow double arrow)/posterior (white double arrow) segment ratio of the eyeball, relative area, number of Erg ECs, and intensities of Tie2, Prox1, and KIf4 immunostaining in CD144+ SC. Dashed lines demarcate the margins of SC. Each area marked by a yellow box is magnified in the corner. Scale bars: $100 \mu \mathrm{m}$. SC area and expression of each molecule in WT mice are normalized to 100\%, and their relative levels in Tiez $2^{\text {iAEC }}$ mice are presented. $n=4-5$ for each group. ${ }^{*} P<0.05$ versus WT by Mann-Whitney $U$ test.

SCs compared with those of WT (Figure 7, A-G). Moreover, the $A 1: A 2^{i \Delta / 4}$ mice had scant GVs, thinned RNFL, and markedly attenuated pSTR and PhNR (Figure 8, A-H), results similar to those of a previous report (26). Of special note, when we deleted both Angpt1 and Angpt2 in 8-week-old $A 1: A 2^{i / 4}$ mice, the mice surprisingly had elevated IOP with a significantly elongated anterior-toposterior segment ratio of the eyeball, markedly reduced area, cellularity, and protein levels of Prox1, Klf4, Tie2, and p-Tie2, and increased $\alpha \mathrm{SMA}^{+}$cells in SC compared with those of WT mice 4 weeks later (Figure 9, A-G). In addition, increased caspase- $3^{+}$ apoptotic ECs were found in SCs of the $A 1: A 2^{i / / 4}$ mice 1 week after tamoxifen administration (Supplemental Figure 7, A-C), suggesting that $\mathrm{SC}$ regression in $A 1: A 2^{i / / 4}$ mice could be mainly attributed to increased apoptosis of ECs. Moreover, the $A 1: A 2^{i / 4}$ mice had a reduced number and diameter of GV, thinned RNFL, and markedly attenuated pSTR and PhNR (Figure 10, A-I). Together, these findings show that deletion of both Angpt1 and Angpt2 from adulthood induces severe $\mathrm{SC}$ regression and phenotypes similar to those in acquired adult-onset POAG.

Throughout the experiments, we detected no noticeable alterations and differences in SCs of the VE-cadherin-Cre-ER ${ }^{\mathrm{T} 2}$ or ubiquitin-Cre-ER ${ }^{\mathrm{T} 2}$ mice prior to tamoxifen administration, with each compared with their respective control WT mice. Moreover, we also detected no noticeable alterations and differences in SCs of the Tie $2^{i A E C}$ or $A 1: A 2^{i \Delta / 4}$ mice prior to tamoxifen administration, with each compared with their respective control WT mice.

Prox1 is also required for adequate SC development and maintenance. We and others recently reported that Prox 1 is expressed in SC ECs $(7,10)$ and that its expression is changed by AHO and VEGFR3 signaling $(8,9)$. To determine the role of Prox1 in SC development, we generated a Prox $1^{\mathrm{i} E \mathrm{CC}}$ mouse by crossing the VE-cadherin-Cre-ER ${ }^{\mathrm{T} 2}$ mouse (34) with the Proxt $\mathrm{fl}^{\text {fll }}$ mouse (39), administered tamoxifen starting at P5, and analyzed them at P7 (Figure 11A). Reduced SC area and diminished Prox1, Klf4, Tie2, and p-Tie 2 in SCs were detected in Prox ${ }^{14 E C}$ mice compared with those of WT mice (Figure 11, B-D). To evaluate the role of Prox1 in SC maintenance, we deleted Prox 1 in SCs starting at 8 weeks old and analyzed them 2 weeks later (Figure 12A) because most of the mice died within 3 weeks, presumably due to severe intestinal edema and hemorrhage. At 2 weeks later, SCs of Prox $1^{\text {iaEC }}$ mice had slightly reduced cellularity and diminished Klf4, Tie2, and p-Tie2 and reduced and disrupted VE-cadherin ${ }^{+}$EC junctions compared with those of WT mice, although there were no differences in IOP, SC area, or number and diameter of GVs between the 2 groups (Figure 12, B-I). Thus, Prox1 plays a substantial role in SC formation, while it plays a minor role in SC maintenance. 
A
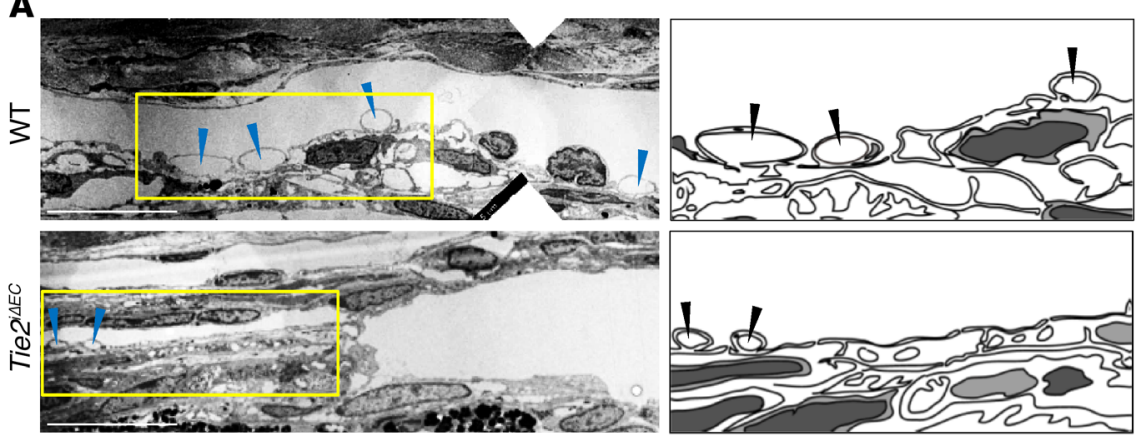

D

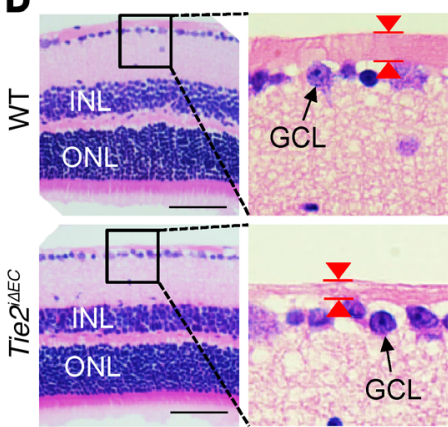

G

WT

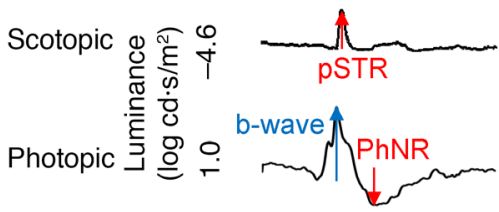

Tubb3

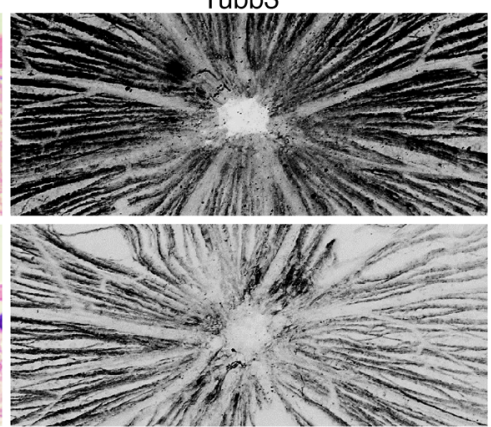

Tie2 $2^{\perp E C}$
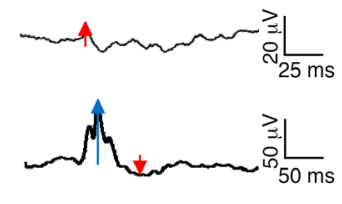

B
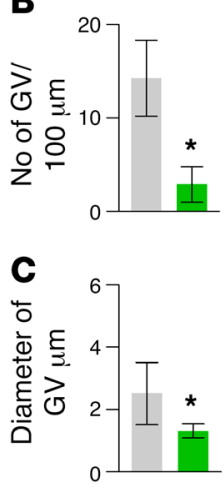

Figure 6. Defective SC integrity by Tie2 depletion impairs AHO and retinal ganglion cell function. (A-C) EM images and comparisons of number and diameter of GVs (blue and black arrowheads) in the inner SC wall. Right panels depict schematic views of yellow-lined boxes. $n=4$ for each group. ${ }^{*} P<0.05$ versus WT by Mann-Whitney $U$ test. Scale bars: $10 \mu \mathrm{m}$. (D-F) Images and comparisons of the thickness (red arrowheads) and Tubb3 distribution of RNFL. Black-lined box is magnified in middle panel. Scale bars: $200 \mu \mathrm{m}$. (G-I) Representative wave responses of electroretinogram and comparisons of pSTR amplitude and PhNR/b-wave amplitude ratio. $n=4$ for each group. ${ }^{*} P<0.05$ versus WT by Mann-Whitney $U$ test.

PROX1 is regulated by TIE 2 in LECs. The aforementioned findings led us to investigate whether a certain molecular cooperation exists among Tie2, AHO, and Prox1 in the formation and maintenance of SC. To gain insight into this question, we performed RNA-sequencing (RNA-seq) analysis in the primarily cultured human dermal LECs (hDLECs) that were incubated with a control siRNA construct (siControl), siTIE2, or siPROX1 (see Methods). Although the transcriptomes of the siTIE2 and siPROX1 gene sets were more similar to each other than to those of the siControl gene set (Supplemental Figure 8A), they displayed more differentially expressed genes (DEGs) than the overlapping genes (Supplemental Figure 8B). Further gene ontology (GO) analysis revealed that the siTIE2 signature was related to defects in the maturation and tubulation of ECs and eNOS signaling, an indicator of flowinduced shear stress (40) (Supplemental Figure 8C and Supplemental Table 3). Meanwhile, the signature of the siPROX1 gene set included defects in the integrity of ECs (Supplemental Figure 8D and Supplemental Table 4). In addition, changes in mRNA and protein expression of hDLECs transfected with siTIE2 (siTIE2LECs) or siControl (siControl-LECs) were validated. In comparison with those of siControl-LECs, siTIE2-LECs displayed reduced
mRNA and protein expression of KLF4, VE-cadherin, and PROX1 (Figure 13, A-D). Moreover, when hDLECs were stimulated with a TIE2 agonistic antibody, ANGPT2-binding and TIE2-activating antibody (ABTAA) (41), TIE2 downstream signals such as AKT and ERK were activated (Figure 13, E and F). Furthermore, ABTAA attenuated IFN- $\gamma$-induced reduction of PROX1 levels in hDLECs, and its effect was abolished by ERK inhibitor, but not AKT inhibitor, indicating that TIE2 activation contributed to upregulating or maintaining PROX1 levels through activation of ERK signaling (Figure 13, G and H). Together, these results implied that interconnected and coordinated roles of TIE2 and PROX1 in SC ECs are required for the formation and maintenance of SC; TIE2 is essential for promoting tubulation and lumen formation for initiation of AHO, which subsequently upregulates PROX1 during SC formation, while it is also indispensable for maintaining SC integrity by keeping adequate $\mathrm{AHO}$ and PROX1 activity.

Reduced Angpts and Tie2, cellularity, cell-cell junction, and transcytosis in aged SC. We next investigated the molecular and cellular changes of aged SC because aging is a major risk factor for adult-onset POAG (42). In particular, AHO resistance between SC and TM increases with age in humans (4), while AHO and lym- 
A

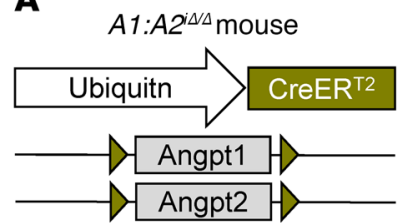

C
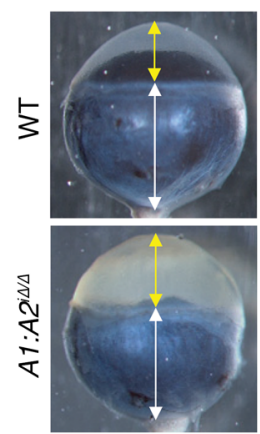

D

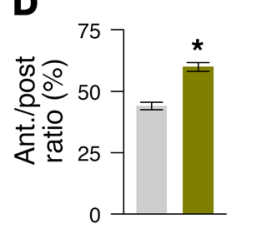

Prox1 CD144
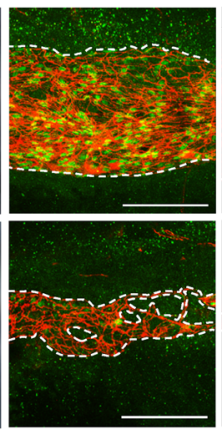

E

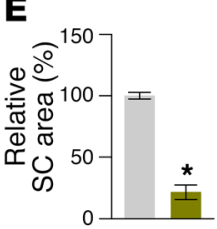

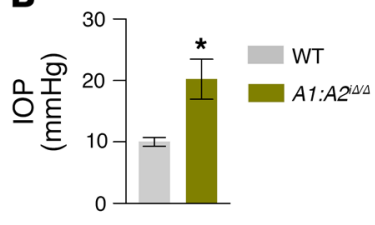

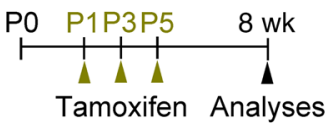

Tamoxifen Analyses

\section{B}
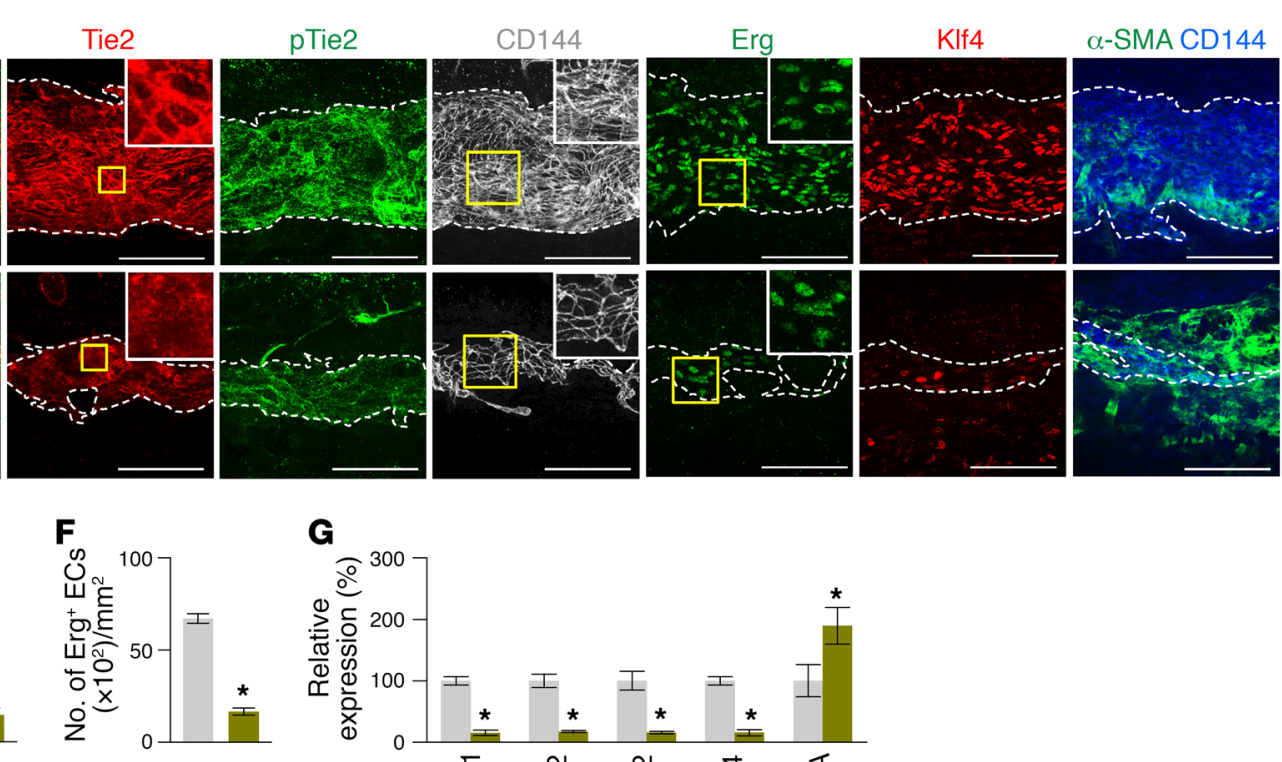

Figure 7. Combined depletion of Angpt1 and Angpt2 impairs SC formation. (A) Diagram for global depletion of Angpt1 and Angpt2 starting at P1 and analyses at 8 weeks after birth using $A 1: A 2^{i \Delta / 4}$ mice. (B-G) Images and comparisons of IOP, an anterior (yellow double arrow)/posterior (white double arrow) segment ratio of the eyeball, relative area, number of Erg' ECs, and intensities of Prox1, Tie2, p-Tie2, KIf4, and $\alpha-S M A$ immunostaining in CD144+ SC. Dashed lines demarcate the margins of SC. Each area marked by a yellow box is magnified in the right top corner. Scale bars: $100 \mu \mathrm{m}$. SC area and expression of each molecule in WT mice are normalized to $100 \%$, and their relative levels in $A 1: A 2^{i \Delta / \Delta}$ mice are presented. $n=4-5$ for each group. ${ }^{*} P<0.05$ versus WT by Mann-Whitney $U$ test.

phatic markers of aged SC are suppressed (8). To clarify whether attenuation of Tie2 is involved in SC aging, we compared the SCs of 2-month-old (adult) mice with those of 18-month-old (aged) mice. In aged mice, reduced Tie 2 and p-Tie2 were found together with reduced Prox1 and cellularity and disorganized VE-cadherin ${ }^{+}$EC junctions, but no change in SC area was identified (Figure 14, A-E). Moreover, reduced number and diameter of GVs were detected in aged mice (Figure 14, A, F, and G), indicating that aged SC has a weakened function in Tie2 signaling and AHO. In addition, compared with adult mice, reduced mRNA and protein levels of Angpt1 and Angpt2 around the SC region were found in aged mice (Figure 14, $\mathrm{H}$ and I). Overall, a reduced Angpt-Tie2 system was detected in SC of aged mice.

Tie2 activation rejuvenates aged SC and rescues impaired SC of $A 1: A 2^{i \Delta / \Delta}$ mice. To test whether Tie2 activation could rejuvenate the aging phenotype of SC, we performed intraocular injection of ABTAA (see Methods) to one eye and an identical injection of Fc to the contralateral eye in the same aged mouse and compared the two eyes (Figure 15A). Although the IOP and SC areas were not significantly altered, the number of $\mathrm{Erg}^{+}$and $\mathrm{Ki}-67^{+}$proliferating ECs, levels of Prox1, Tie2, p-Tie2, and Klf4, and number and diameter of GVs in SCs were significantly increased in the ABTAA-treated eye compared with the Fc-treated eye (Figure 15, B-J). In contrast, no differences in IOP, SC area, and Prox1 between ABTAA-treated and Fc-treated eyes were found in the Tie ${ }^{i \Delta E C}$ mice (Supplemental Figure 9, A-E), indicating that the action of ABTAA is solely mediated through Tie2. We also evaluated the effect of intraocular injection of ABTAA on the SC of $A 1: A 2^{i \Delta / \Delta}$ mice (Figure 16A). At 2 weeks after injection, decreased IOP together with increased SC area and levels of Prox1 and Tie2 were detected in the ABTAA-treated eye compared with the Fc-treated eye (Figure 16, B-E). Together, these findings suggest that Tie 2 activation rejuvenates aged SC and rescues the impaired SC of $A 1: A 2^{i \Delta / \Delta}$ mice.

\section{Discussion}

The most intriguing finding of this study is the identification of a critical role of the Angpt-Tie2 system in maintaining SC integrity (Figure 17). Impairment of this system could be involved in adult-onset POAG, which is the most common type of glaucoma in patients. Of importance, Tie2 is highly present in SC ECs in adults and supposedly decreases with aging, which is an important risk factor for POAG. Worthy of special note, Tie2 activation can recover the phenotypes of POAG in double Angpt1/Angpt2deleted mice and can rejuvenate aged SC. 
A

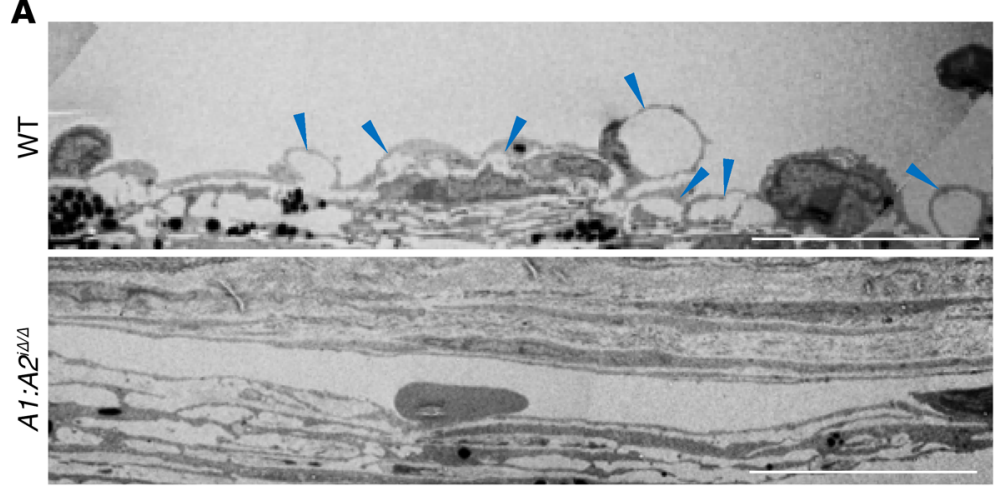

C

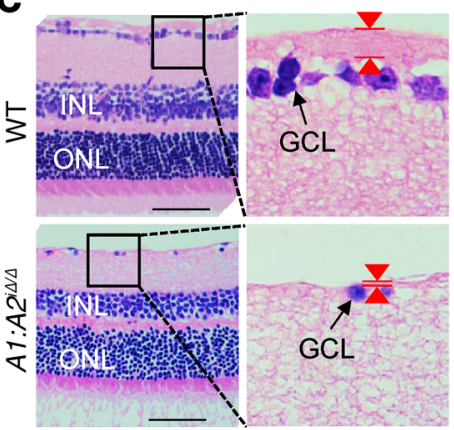

$\mathbf{F}$

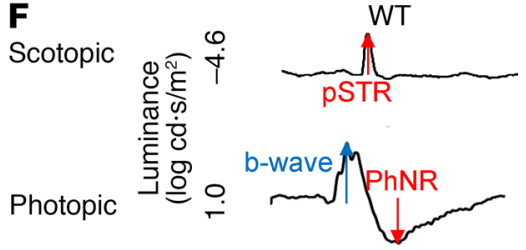

Tubb3

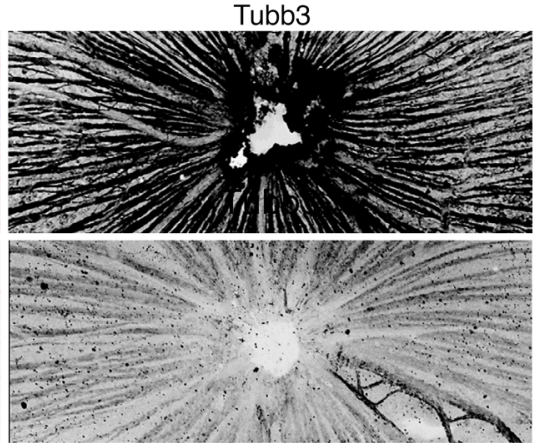

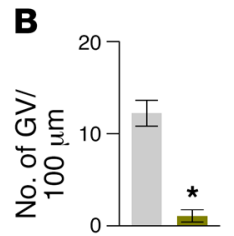

D

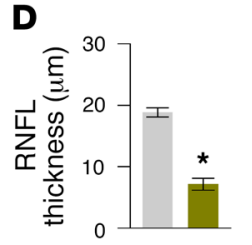

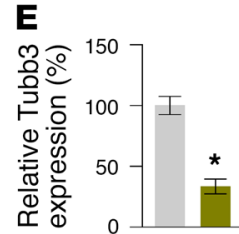

Figure 8. Impaired SC formation in A1:A2 ${ }^{i / 4}$ mice leads to glaucoma. (A and B) EM images and comparisons of the number of GVs (blue arrowheads) in the inner SC wall. Scale bars: $10 \mu \mathrm{m} . n=4$ for each group. ${ }^{*} P<0.05$ versus WT by Mann-Whitney $U$ test. (C-E) Images and comparisons of thickness (red arrowheads) and Tubb3 distribution of RNFL. Black-lined box is magnified in middle panel. Scale bars: $200 \mu \mathrm{m}$. (F-H) Representative wave responses of electroretinogram and comparisons of pSTR amplitude and PhNR/b-wave amplitude ratio. $n=4$ for each group. ${ }^{*} P<0.05$ versus WT by Mann-Whitney $U$ test.

Abnormally high resistance against AHOc through SC is a major causative factor in the pathogenesis of glaucoma, especially for POAG (5). When SC is impaired, AH drainage is inhibited and IOP is increased, ultimately leading to glaucoma. In parallel, when there is an impairment in peripheral LVs, drainage of interstitial fluid is inhibited, ultimately leading to lymphedema. In this regard, glaucoma induced by impaired SC could be considered as a sort of "eye lymphedema." Our findings highlight SC as a functional reservoir of $\mathrm{AHO}$ as well as a main structure for glaucomagenesis when severely impaired. Although it has been long debated (43-45), recent studies indicate that AHOc, rather than $\mathrm{AHOu}$, is a primary route for AHO in C57BL/6J mice $(46,47)$, similar to that in humans. We note that a more than $50 \%$ reduction in SC area and a marked reduction in transcytotic activity in SC ECs are prerequisites for the significant elevation of IOP, retinal neuronal damage, and impairment of retinal ganglion cell function. This phenomenon could be explainable based on a previous report (48) showing SC elasticity in tolerating increased resistance to AHOc to a certain degree. In fact, patients with POAG have markedly reduced SC dimensions, which are correlated with increased outflow resistance (49-52), emphasizing a significance for SC in POAG pathogenesis. Moreover, GVs, which are formed in response to a pressure gradient between IOP and episcleral venous pressure and transport $\mathrm{AH}$, are rarely found in the ECs of the inner SC wall of POAG patients (53), reflecting severely reduced flow through AHOc. In this regard, pathogenesis of POAG is similar to pathogenesis in adult-onset glaucomatous phenotypes of Tie $2^{i \Delta E C}$ or $A 1: A 2^{i \Delta / \Delta}$ mice.

This study demonstrates that the pericyte-derived Angpt1 and the endothelium- and TM-derived Angpt2 play overlapping and profound roles through Tie 2 signaling not only in the generation, but also in the maintenance of SC. In this regard, transcriptional regulation of Angpt1 and Angpt2 during SC generation and maintenance needs to be further elucidated. While Tie 2 deletion and double Angpt1/Angpt2 deletion induced severe glaucomatous phenotypes with severe regression of SC, deletion of either Angpt1 or Angpt2 did not result in elevated IOP despite mild SC regression. In this regard, Angpt 2 seems to play an agonistic rather than antagonistic role for Tie 2 in the generation and maintenance of SC. Intriguingly, we found a significant downregulation of Angpt 2 in the TM of 24 individuals with POAG compared with that of 26 control subjects in the profiled human metadata (data from NCBI's Gene 

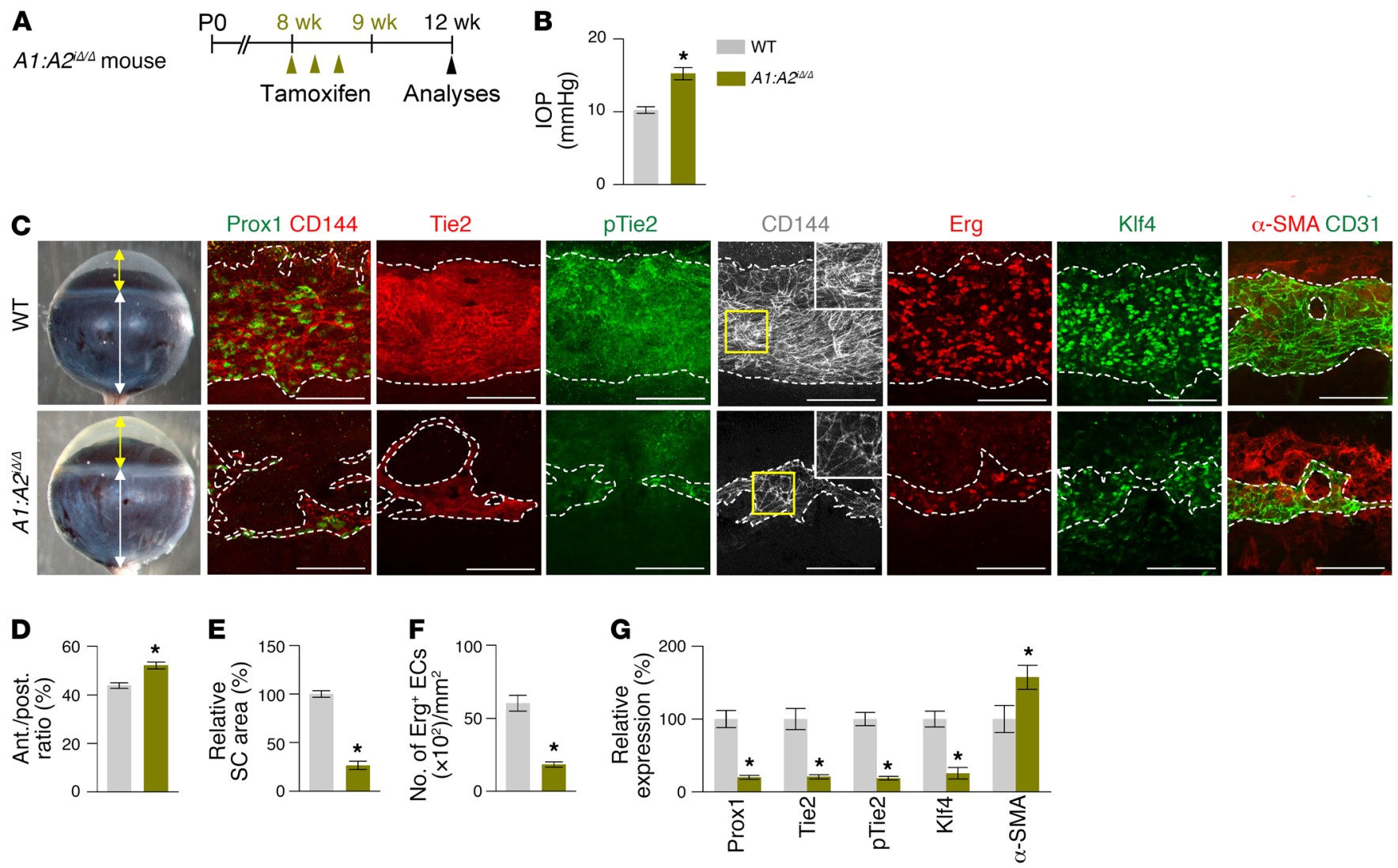

Figure 9. Combined depletion of Angpt1 and Angpt2 in adult mice causes SC regression. (A) Diagram for combined global depletion of Angpt1 and Angpt2 in 8-week-old mice and analyses 4 weeks later using $A 1: A 2^{i / 4}$ mice. (B-C) Images and comparisons of IOP, an anterior (yellow double arrow)/posterior (white double arrow) segment ratio of the eyeball, relative area, number of Erg ECs, and intensities of Prox1, Tie2, p-Tie2, KIf4, and $\alpha$-SMA immunostaining in $\mathrm{CD}_{144^{+}}$or CD31+ SC. Dashed lines demarcate the margins of SC. Each area marked by a yellow box is magnified in the corner. Scale bars: $100 \mu \mathrm{m}$. SC area and expression of each molecule in WT mice are normalized to $100 \%$, and their relative levels in $A 1: A 2^{i / 4}$ mice are presented. $n=4-5$ for each group. ${ }^{*} P<0.05$ versus $W T$ by Mann-Whitney $U$ test.

Expression Omnibus; GEO GSE27276, GSE4316 and GSE27058), supporting our hypothesis. Our detailed analyses indicate that Tie2 activation is definitely required for formation of the canal tube and lumen by differentiation and maturation of SC progenitor cells for initiation of AHO, which subsequently upregulates expression of Prox1 and Klf4 during SC development. In addition, transcriptional activity of Prox 1 is also required for differentiation and maturation of SC and upregulation of Tie 2 and Klf4 expression during SC development. However, while continuous Angpt-Tie2 signaling and $\mathrm{AHO}$ are definitely required to maintain Tie2 activation and SC integrity (8), the transcriptional activity of Prox1 seems somewhat redundant. Based on these findings, an interconnected pathway of Tie2-AHO-Prox1 could be proposed as an essential loop for SC formation and maturation, while only a biconnected pathway between Tie2 and AHO could be proposed as a critical loop for the maintenance of SC integrity (Figure 17). Indeed, reduced AngptTie2 signaling not only leads to defective SC development, but also impedes maintenance of SC integrity, leading to compromised $\mathrm{AH}$ drainage. In this respect, Tie2 activation could be a potential therapeutic approach for ameliorating glaucoma progression through recovery of SC integrity and drainage function and subsequent IOP reduction (Figure 17). Indeed, we demonstrate that Tie2 activation through intraocular administration of ABTAA reduces the elevated
IOP and recovers SC regression in double Angpt1/Angpt2-deleted mice. This finding was in parallel with a previous report about the substantial role of VEGFC-VEGFR3 signaling in rejuvenating aged SC (9). In addition, considering that VEGFC-VEGFR3 signaling plays an essential role in SC formation $(8,9)$ and that Angpt-Tie2 signaling shares this role, it is supposed that there is a close crosstalk between those signaling pathways in SC.

Cellular senescence of SC is associated with accumulation of harmful stimuli and reactive oxygen species, which may cause dysfunctional drainage in humans and mice $(39,54)$. Along with the previous report demonstrating decreased $\mathrm{AHO}$ and reduced expression of lymphatic markers such as Prox1 and VEGFR3 in aged SC (8), we reveal here that aged SC ECs exhibit attenuated intercellular junctions and decreased cellularity and transcytosis together with an attenuated Angpt-Tie2 system. However, there was no significant difference in IOP between young adult and aged mice. We postulate that this is because, as with human eyes, $\mathrm{AH}$ formation rate is decreased by about $40 \%$ in aged mice compared with young adult mice (46); thus, resistance to AHOc is not proportionally elevated with regressing SC in aged animals. Nevertheless, given that Tie 2 activation apparently rejuvenates aged SC, intra-ocular administration of Tie2 agonist could be one of therapeutic options for treating patients with age-dependent POAG. 
A

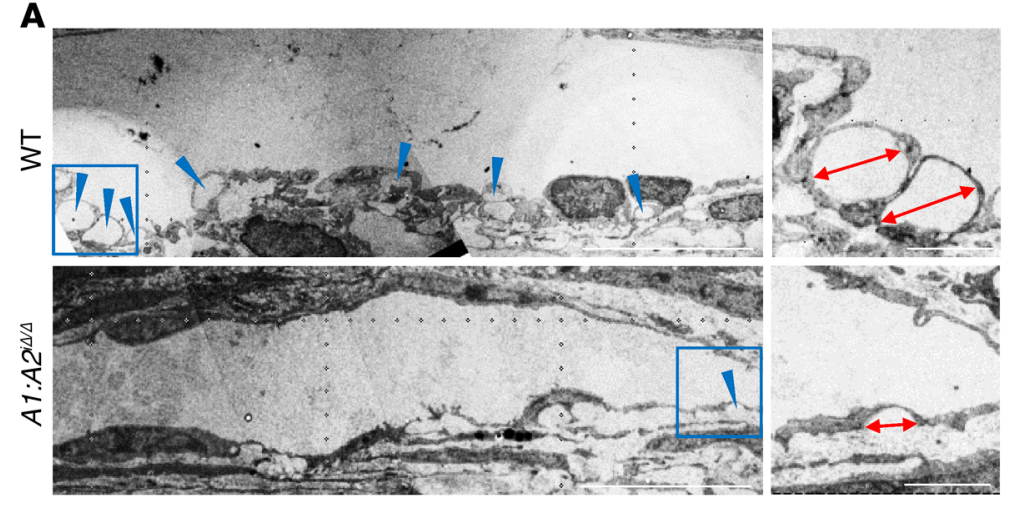

B

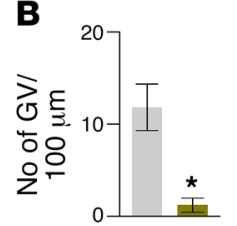

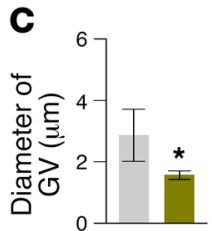

D
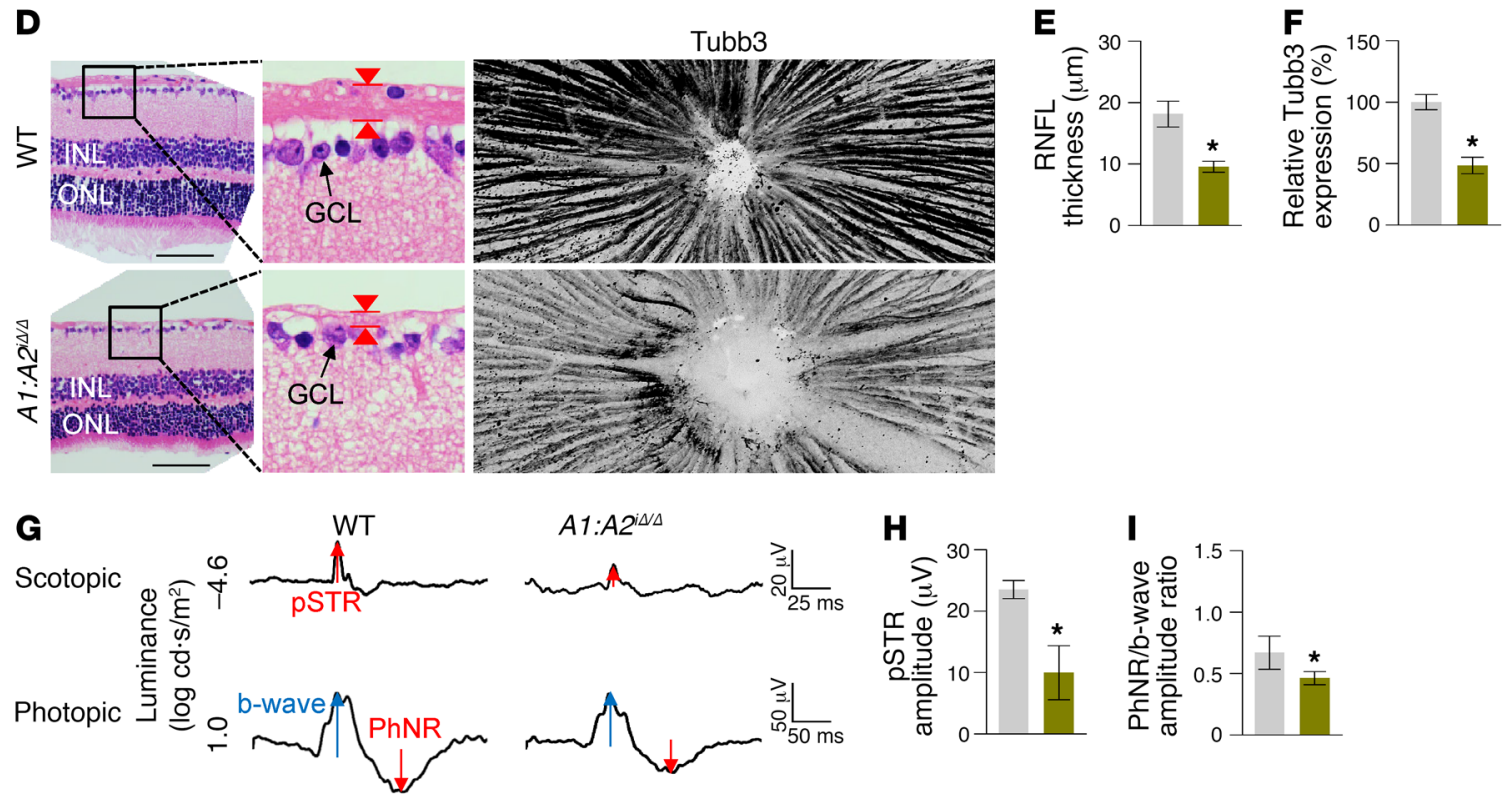

Figure 10. SC regression in A1:A2 ${ }^{i / 4}$ mice leads to adult-onset glaucoma. (A-C) EM images and comparisons of the number and diameter of $\mathrm{GVs}$ (blue arrowheads) in the inner SC wall. Blue-lined box is magnified in right panel. Red double arrows indicate diameters of GV. Scale bars: $10 \mu \mathrm{m}$ (left panels); 2 $\mu \mathrm{m}$ (right panels). $n=4$ for each group. ${ }^{*} P<0.05$ versus WT by Mann-Whitney $U$ test. (D-F) Images and comparisons of thickness (red arrowheads) and Tubb3 distribution of RNFL. Black-lined box is magnified in middle panel. Scale bars: $200 \mu \mathrm{m}$. (G-I) Representative wave responses of electroretinogram and comparisons of pSTR amplitude and PhNR/b-wave amplitude ratio. $n=4$ for each group. ${ }^{*} P<0.05$ versus WT by Mann-Whitney $U$ test.

Even though several pharmacological agents are widely used as first-line therapy for treating POAG, there are important unmet medical needs. While some agents, including $\beta$-adrenergic receptor blockers, $\alpha 2$-adrenergic receptor agonists, and carbonic anhydrase inhibitors, decrease production of $\mathrm{AH}$, others, such as prostaglandin analogues, are mostly focused on promoting AHO through AHOu while minimally affecting AHOc (2). Thus, these current therapeutic options are only temporary measures and not fundamental solutions because AHOc is the major AHO pathway (4), and impeded AHO through this pathway is the main factor underlying elevated IOP in POAG (5). In this regard, facilitating AHO through Tie 2 activation-induced recovery of SC could be a potential avenue for patients with POAG.

Together, the results of this study delineate the critical role of an integrated network consisting of Tie2, AHO, and Prox1 in development and maintenance of SC and identify the crucial role of Angpt-Tie2 signaling in SC maintenance in the context of POAG, providing decisive clues regarding its pathogenesis and treatment.

\section{Methods}

Mice. Specific pathogen-free (SPF) C57BL/6J mice (catalog 000664), Tie2-GFP mice (catalog 003658), and UBC-Cre-ER ${ }^{\mathrm{T} 2}$ mice (catalog 007001) were purchased from the Jackson Laboratory. Angpt $t^{\Re / / l}$ mice were a gift from Yoshikazu Nakaoka (Osaka University, Osaka, Japan), Angpt1-GFP mice were a gift from Sean J. Morrison (University of Texas Southwestern, Dallas, Texas, USA), Angpt2-lacZ mice were a gift from Nicholas Gale (Regeneron Pharmaceuticals), and $V e g f 2^{2 / f l l}$ mice were a gift from Masanori Hirashima (Kobe University, Kobe, Japan).

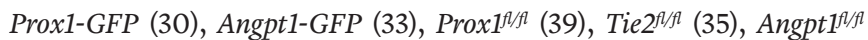
(36, 37), Angpt $2^{R / f l}$ (38), Vegfr2 ${ }^{R / f l}$ (55), and Angpt2-lacZ (22) mice were transferred and bred in our SPF animal facility at KAIST. To deplete Prox1, Tie2, or Vegfr2 genes specifically in ECs, VE-cadherin-Cre-ER ${ }^{\mathrm{T} 2}$

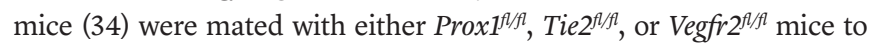
obtain EC-specific Prox1-, Tie2-, or Vegfr2-deleted mice, respectively, in a tamoxifen-dependent manner. To delete Angpt1 or Angpt2 globally, Angpt $t^{A / / R}$ or Angpt $2^{R / / A}$ mice were crossed with the UBC-Cre-ER ${ }^{\mathrm{T} 2}$ mouse. Tamoxifen (2 mg, T5648, Sigma-Aldrich) was injected intraperitoneally a total of 3 times every 2 days into the 8 -week-old genetically modified 
A

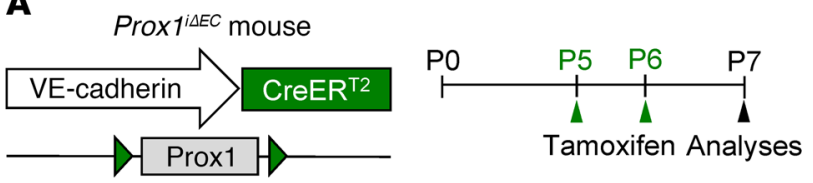

B
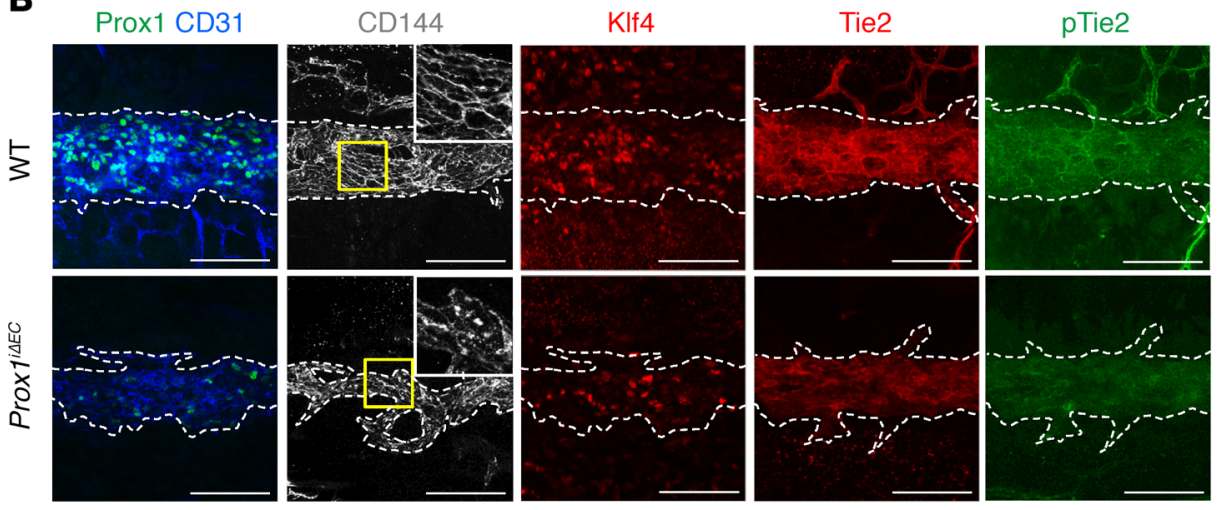

Figure 11. Prox1 is required for SC development. (A) Diagram for EC-specific depletion of Prox 1 in SC starting at P5 and analyses at P7 using Prox $7^{\text {isEC }}$ mice. (B-D) Images and comparisons of relative area of SC and intensities of Prox1, KIf4, Tie2, and p-Tie2 immunostaining in $\mathrm{CD} 31^{+}$or $\mathrm{CD} 144^{+} \mathrm{SC}$. Dashed lines demarcate the margins of SC, and each area marked by a yellow box is magnified in the top corner. Scale bars: 100 $\mu \mathrm{m}$. SC area and expression of each molecule in WT group are normalized to $100 \%$, and their relative levels in Prox ${ }^{i \Delta E C}$ mice are presented. $n=4$ for each group. ${ }^{*} P<0.05$ versus WT by Mann-Whitney $U$ test.

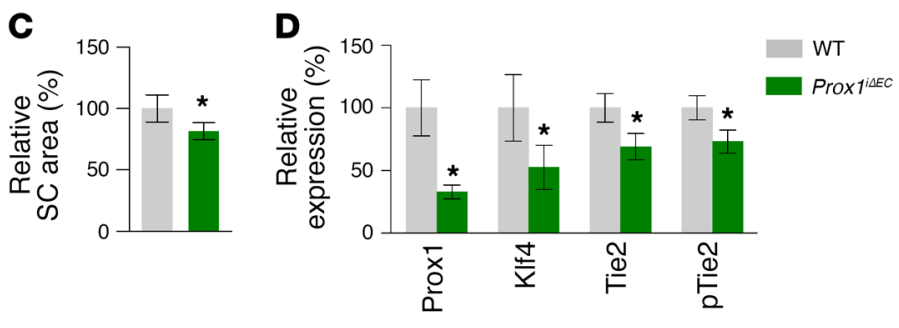

mice. For postnatal mice, except $\operatorname{Prox} 1^{f / f l}$ mice, $50 \mu \mathrm{g}$ 4-hydroxytamoxifen (H7904) was injected subcutaneously every 2 days from P1 to P5. $\operatorname{Prox}^{f / f l}$ mice intercrossed with VE-cadherin-Cre-ER ${ }^{\mathrm{T} 2}$ mice were injected with 4-hydroxytamoxifen daily from P5 to P6 due to early lethality. All mice were fed with free access to a standard diet (PMI LabDiet) and water. Mice were anesthetized with an intraperitoneal injection of $40 \mathrm{mg} / \mathrm{kg}$ ketamine and $5 \mathrm{mg} / \mathrm{kg}$ xylazine before any procedures.

Sampling of human corneas. Human corneal samples were obtained from the peripheral corneoscleral rim of healthy cadaveric donor tissue (Lions Eye Bank, Portland, Oregon, USA) left over from penetrating keratoplasty at the Samsung Medical Center. These were exempted from the formal review of the IRB at Samsung Medical Center due to the lack of patient identifiers and the intention of being otherwise discarded. We examined the protein expression of TIE2 and PROX1 in SC of these samples using immunofluorescence (IF) staining.

Histological analyses. IF staining for whole-mounted and sectioned corneas and retinas were performed as previously described (8). Briefly, enucleated eyes were first fixed with $4 \%$ paraformaldehyde (PFA) in PBS at room temperature (RT) for 20 minutes (for adult mice) or $2 \%$ PFA in PBS at $4^{\circ} \mathrm{C}$ for 30 minutes (for postnatal mice). For IF staining of sectioned corneas, fixed eyeballs were then dehydrated in $20 \%$ sucrose solution overnight, embedded in tissue-freezing medium (Leica), and cut into $14-\mu \mathrm{m}$ sections. For IF staining of whole-mounted retina, after isolating the retina from eyeball, the retinas were additionally fixed with 1\% PFA in PBS at RT for 1 hour. Samples were blocked with $5 \%$ donkey or goat serum in PBST (0.3\% Triton X-100 in PBS) and then incubated in blocking solution with one or more of the following antibodies at $4^{\circ} \mathrm{C}$ overnight: anti-CD31 (hamster monoclonal, clone $2 \mathrm{H} 8$, catalog MAB1398Z, Millipore); anti-collagen type IV (rabbit polyclonal, catalog LSL-LB-1407, Cosmo Bio); Cy3-conjugated anti-F-actin (catalog A12379, Thermo Fisher Scientific); anti-Prox1 (rabbit polyclonal, catalog 102-PA32AG, ReliaTech); anti-Prox1 (goat polyclonal, catalog AF2727, R\&D systems); anti-VE-cadherin (CD144) (rat monoclonal, clone 11D4.1, catalog 550548, BD Biosciences); anti-Tie2 (goat polyclonal, catalog AF762, R\&D Systems); anti-p-Tie2 (rabbit polyclonal, catalog AF2720, R\&D systems); anti-Klf4 (goat polyclonal, catalog AF3158, R\&D Systems); anti-Erg (rabbit polyclonal, catalog SC-353, Santa Cruz Biotechnology Inc.); FITC- or Cy3-conjugated antia-SMA (mouse monoclonal, clone 1A4, catalog F3777/catalog C6198, Sigma-Aldrich); anti-LYVE-1 (rabbit polyclonal, catalog 11-034, AngioBio); anti-VEGFR3 (goat polyclonal, catalog AF743, R\&D Systems); anti-VEGFR2 (goat polyclonal, catalog AF644, R\&D Systems); anti-Ki67 (rabbit monoclonal, clone SP6, catalog ab16667, Abcam); anti-Angpt2 (human monoclonal, clone 4H10) (41); anti-PDGFR $\beta$ (rat monoclonal, clone APB5, catalog NC0091961, eBioscience); anti-Tubb3 (rabbit polyclonal, catalog PRB-435P, BioLegend); and anti-cleaved caspase-3 (rabbit polyclonal, catalog 9661, Cell Signaling Technology). Following several washes, the samples were incubated at RT for 4 hours with the following secondary antibodies: FITC-, Cy3- or Cy5-conjugated anti-hamster IgG, anti-rabbit IgG, anti-rat IgG, antigoat IgG, and anti-human IgG antibody (Jackson ImmunoResearch,). For whole-mounted corneas or retinas, the samples were cut radially and then mounted in fluorescent mounting medium (Vector or Dako).

To evaluate $\beta$-gal activity, the corneas were incubated with a staining solution $(5 \mathrm{mM}$ potassium ferricyanide, $2 \mathrm{mM}$ magnesium chloride, $5 \mathrm{mM}$ potassium ferrocyanide, and $1 \mathrm{mg} / \mathrm{ml}$ 4-chloro-5-bromo- 

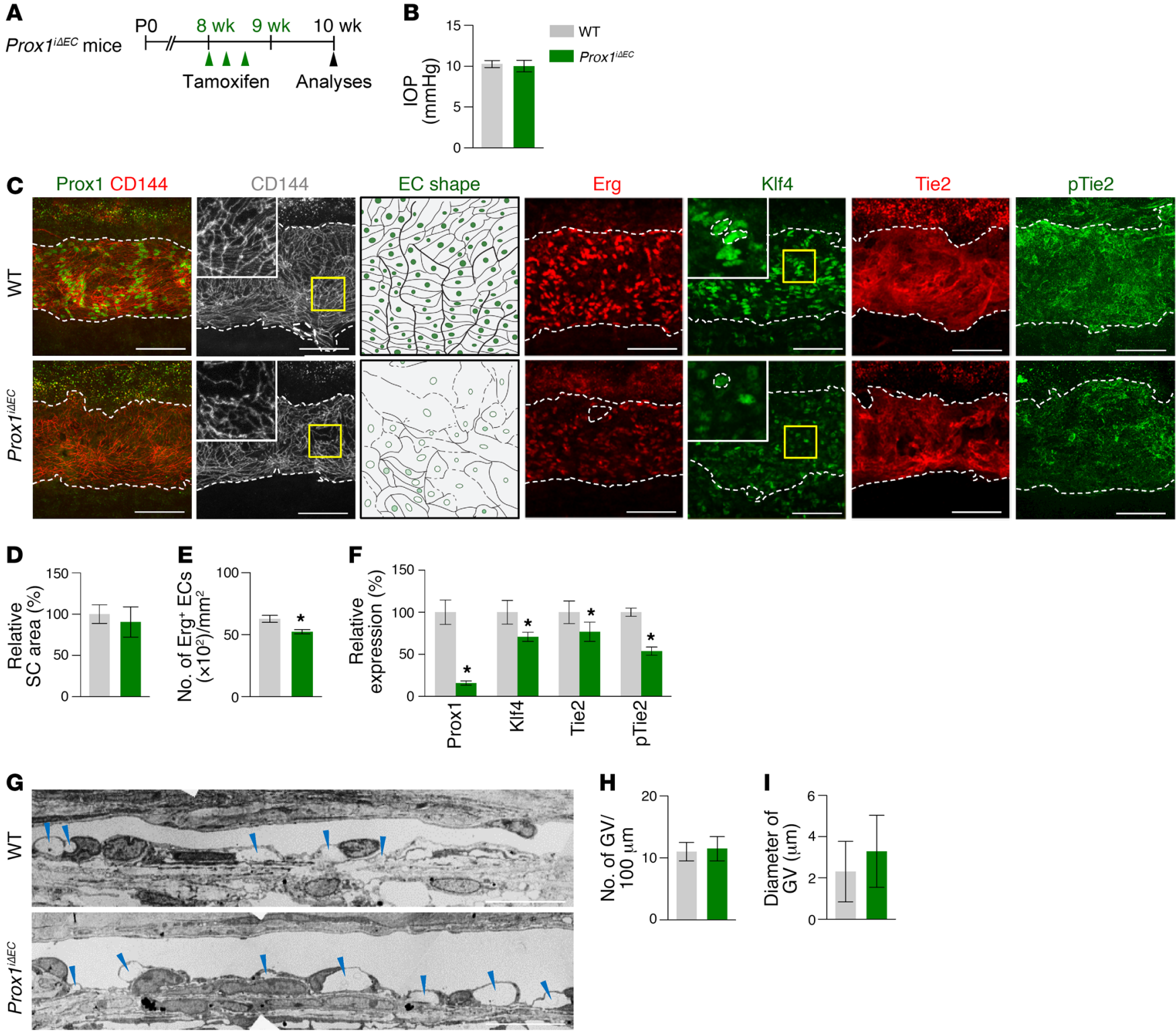

Figure 12. Prox1 is required for SC maintenance. (A) Diagram for EC-specific depletion of Prox 1 in SCs in 8-week-old mice and analyses 2 weeks later using Prox $1^{i 4 E C}$ mice. (B-F) Images and comparisons of IOP, relative area, number of Erg ECs, and intensities of Prox1, KIf4, Tie2, and p-Tie2 immunostaining in $\mathrm{CD}_{144}{ }^{+}$SC. Dashed lines demarcate the margins of SC, and each area marked by a yellow box is magnified in the top corner. Scale bars: $100 \mu \mathrm{m}$. SC area and

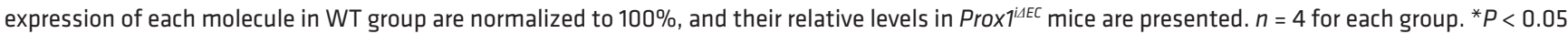
versus WT by Mann-Whitney $U$ test. (G-I) EM images and comparisons of the number and diameter of GVs (blue arrowheads) in the inner SC wall. $n=4$ for each group. Scale bar: $10 \mu \mathrm{m}(\mathbf{G})$.

3-indolyl- $\beta$-D-galactopyranoside [X-gal] in PBS) at $37^{\circ} \mathrm{C}$ for 12 hours. SA- $\beta$-gal activity was examined with the Senescence Detection Kit (Cell Signaling Technology) according to the manufacturer's instructions. All immunofluorescent imaging procedures were performed using a Zeiss LSM 780 or 880 confocal microscope equipped with argon and helium-neon lasers (Carl Zeiss). The images of $\beta$-gal activity were acquired with a microscope equipped with a CCD camera (Carl Zeiss). For H\&E staining, retinas were fixed overnight in $4 \%$ PFA. After tissue processing using standard procedures, samples were embedded in paraffin and cut into 5 - $\mu \mathrm{m}$ sections through the center of the eye (determined by the presence of the optic disc and optic nerve), followed by H\&E staining. Standard light microscopy (Axio Zoom.
V16, Carl Zeiss) was performed to measure RNFL thickness, and retinal sections were photographed at both the left and right points approximately $500 \mu \mathrm{m}$ away from the center of the optic nerve head.

Morphometric analyses. Morphometric analyses of SC, cornea, and retina were performed using ImageJ software (NIH) or ZEN 2012 software (Carl Zeiss). The relative area of SC was calculated as a percentage of the $\mathrm{CD} 144^{+}$or $\mathrm{CD} 31^{+}$area divided by its control area. To determine the area of limbal LVs and BVs, Prox $1^{+}$areas and CD $144^{+}$ areas were measured. To quantify the relative expression of Prox1 and $\mathrm{Klf} 4$, intensities were measured in the nucleus region of $\mathrm{CD} 144^{+}$or CD $31^{+}$SC. To quantify the expression of Tie2, p-Tie2, VEGFR2, and VEGFR3, intensities were measured in the $\mathrm{CD} 144^{+}$or $\mathrm{CD} 31^{+} \mathrm{SC}$ area. 

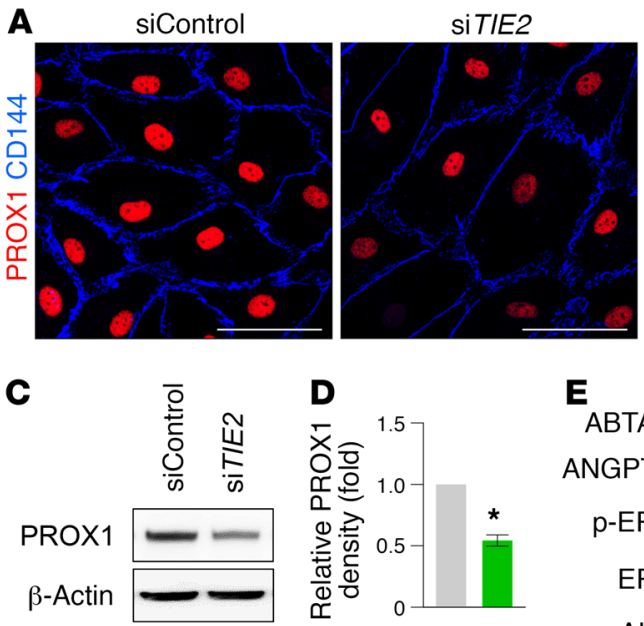

D

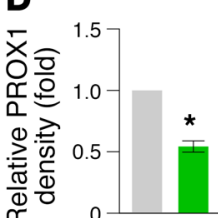

E
B

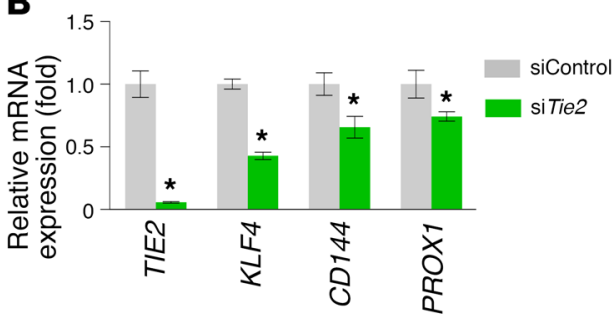
ABTAA ANGPT2

p-ERK

ERK p-AKT p-AKT
$\mathbf{F}$

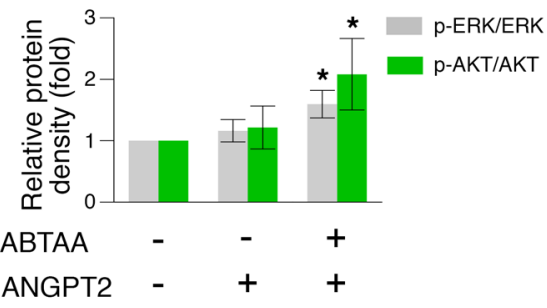

G

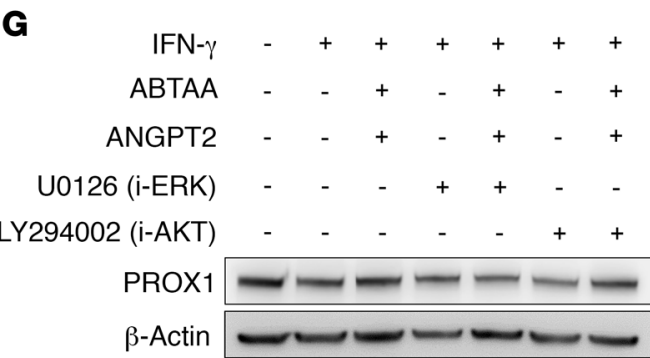

H

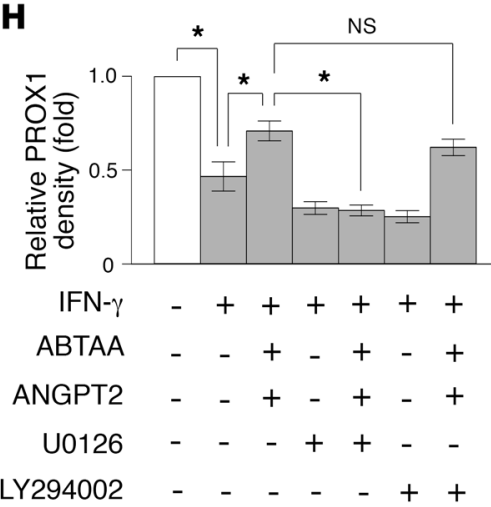

Figure 13. PROX1 expression in hDLECs is regulated by ANGPT-TIE2 signaling. (A) Images showing PROX1 (red) and CD144 (blue) in siControl-LECs and siTIE2-LECs. Scale bars: $50 \mu \mathrm{m}$. (B) Comparison of TIE2, KLF4, CD144, and PROX1 mRNA expression in siControl-LECs and siTIE2-LECs. Fold changes in mRNA expression relative to the levels of siControl-LECs are presented. $n=4$ for each group. ${ }^{*} P<0.05$ versus siControl-LECs by Mann-Whitney $U$ test. (C and D) Immunoblot detection of PROX1 protein in siControl-LECs and siTIE2-LECs. Densitometric analysis of the relative level of PROX1 is shown.

$n=5$ for each group. ${ }^{*} P<0.05$ versus siControl-LECs by Mann-Whitney $U$ test. (E and $\mathbf{F}$ ) Immunoblot detection of TIE2 downstream signaling proteins ERK, $p$-ERK, AKT, and $p$-AKT in hDLECs stimulated with ABTAA. Densitometric analyses of the $p$-ERK/ERK ratio and $p-A K T / A K T$ ratio are shown. $n=5$ for each group. ${ }^{*} P<0.05$ by Kruskal-Wallis test followed by Tukey's HSD test with ranks. (G and $\left.\mathbf{H}\right)$ Immunoblot images showing modulation of PROX1 expression in hDLECs cultured with or without ABTAA and with or without inhibitors of ERK or AKT pathways. Densitometric analysis of the relative level of PROX1 is shown. $n=5$ for each group. ${ }^{*} P<0.05$ by Kruskal-Wallis test followed by Tukey's HSD test with ranks.

The number of $\mathrm{Erg}^{+}, \mathrm{Ki}-67^{+}$, and caspase- $3^{+}$ECs were counted within the $\mathrm{CD} 144^{+}$or $\mathrm{CD} 31^{+} \mathrm{SC}$ area of the random 0.0558 or $0.0803 \mathrm{~mm}^{2}$ area. The expression of $\alpha$-SMA on the outer wall of the SC was calculated as a percentage of positive area divided by $\mathrm{CD} 144^{+}$or $\mathrm{CD} 31^{+}$ $\mathrm{SC}$ area. In order to measure the expression of Tubb3 in retinal nerve fiber, intensities were calculated in the Tubb3 ${ }^{+}$RNFL and divided by retinal area. All measurements of SC were performed in all quarters of the anterior segment in each eye, excluding the portion of the bifurcation of LPCAs, unless indicated otherwise. For statistical analysis, the values from all quarters in each eye were averaged. For comparison of staining intensities, the values were normalized by the background signals in nonvascularized regions, and their ratios were normalized by control and presented as percentages.

$E M$. To capture ultrastructure EM images of SC, eyeballs were sectioned after perfusion-fixation with $4 \%$ PFA and $0.25 \%$ glutaraldehyde in $0.1 \mathrm{M}$ phosphate buffer ( $\mathrm{pH}$ 7.4). Samples were then fixed overnight in
$2.5 \%$ glutaraldehyde, post-fixed with $1 \%$ osmium tetroxide, and dehydrated with a series of increasing ethanol concentrations followed by resin embedding. Ultrathin sections $(70 \mathrm{~nm})$ were obtained with an ultramicrotome (UltraCut-UCT, Leica) and were then collected on copper grids. After staining with $2 \%$ uranyl acetate and lead citrate, samples were examined by transmission EM (Tecnai G2 Spirit Twin, FEI) at $120 \mathrm{kV}$.

Electroretinogram. To assess the functionality of inner retina at indicated time points, a full-field electroretinogram was recorded in the indicated mice using the Phoenix Micron IV System (Phoenix Research Labs). Mice were dark or light adapted for 12 hours prior to electroretinogram monitoring, then were anesthetized and placed on a heating pad to maintain body temperature. After pupil dilatation by 1-time topical application of $0.5 \%$ tropicamide/0.5\% phenylephrine mixed eye drop (Mydrin-P, Santen), the cornea was located by gold-plated objective lens, and silver-embedded needle electrodes were located at the forehead (reference) and tail (ground). Using LabScribeERG software 
A
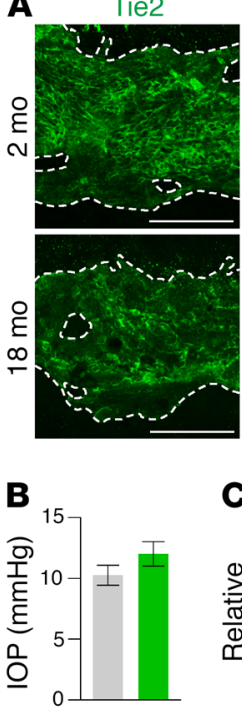
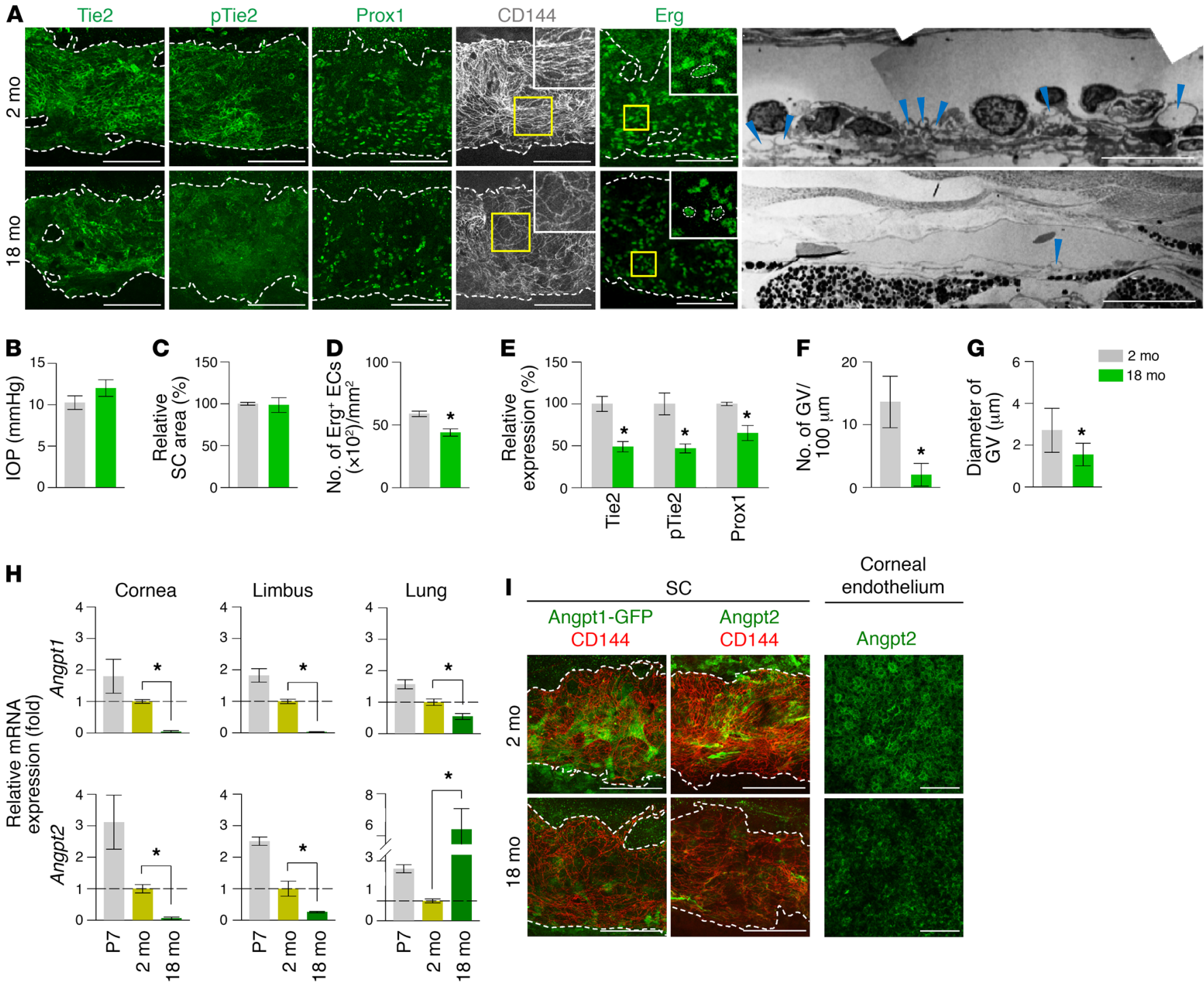

$\mathrm{SC}$
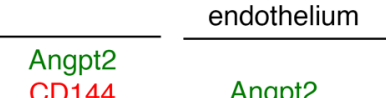

Figure 14. Reduced Angpts and Tie2, cellularity, cell-cell junction, and transcytosis in aged SC. (A-E) Images and comparisons of IOP, relative area, number of Erg' ECs, and intensities of Tie2, p-Tie2, and Prox1 immunostaining in CD144+ SC of 2-month-old versus 18-month-old mice. Dashed lines demarcate the margins of SC. Each area marked by a yellow box is magnified in the top corner. Scale bars: $100 \mu \mathrm{m} ; 10 \mu \mathrm{m}$ (EM). SC area and expression of each molecule in 2-month-old mice are normalized to $100 \%$, and their relative levels in 18 -month-old mice are presented. $n=5$ for each group. ${ }^{*} P<0.05$ versus 2 months by Mann-Whitney $U$ test. (A, F, and $\mathbf{G}$ ) EM images and comparisons of the number and diameter of GV (blue arrowheads) in the inner SC wall. $n=4$ for each group. ${ }^{*} P<0.05$ versus 2 months by Mann-Whitney $U$ test. $(\mathbf{H})$ Comparison of Angpt 1 and Angpt2 mRNA expression in cornea, limbus, and lung of mice at P7, 2 months, and 18 months of age. Fold changes in mRNA expression relative to the levels of 2-month-old mice are presented. $n=4$ for each group. ${ }^{*} P<0.05$ by Kruskal-Wallis test followed by Tukey's HSD test with ranks. (I) Images showing expression of Angpt1, Angpt2, and CD144 in SC and Angpt2 in corneal endothelium. Scale bars: $100 \mu \mathrm{m}$.

(Version 3, Phoenix Research Labs), the stimulus and recording of electroretinogram were performed according to the manufacturer's instructions. To obtain PhNR and the b-wave of the photopic electroretinogram (light-adapted conditions), a digital bandpass filter ranging from 2 to $200 \mathrm{~Hz}$ and stimuli ranging from 0.4 to $2.2 \mathrm{log}\left(\mathrm{cd} \cdot \mathrm{sec} / \mathrm{m}^{2}\right)$ with $1.3 \mathrm{log}$ $\left(\mathrm{cd} \cdot \mathrm{sec} / \mathrm{m}^{2}\right)$ background were used. To yield pSTR of the scotopic electroretinogram (dark-adapted condition), filters ranging from 0.05 to 300 $\mathrm{Hz}$ and stimuli ranging from -5.5 to $-3.0 \mathrm{log}\left(\mathrm{cd} \cdot \mathrm{sec} / \mathrm{m}^{2}\right)$ were used. After averaging the signals, the amplitude was presented by LabScribeERG software and used for analyses.

IOP measurement. IOP measurements were performed with a rebound tonometer (TonoLab, Tiolat) as previously described (56).
IOP was measured by placing the tip of the pressure sensor approximately $1 / 8$ inch from the central cornea immediately after anesthetizing the mice. The digital readouts of 5 consecutive IOP measurements were acquired from the tonometer.

Intraocular administration. Using a glass capillary pipettefitted Nanoliter 2000 Microinjector (World Precision Instruments), approximately $1 \mu \mathrm{l}$ PBS containing the premixed Angpt2 $(0.01 \mu \mathrm{g})$ and ABTAA $(5 \mu \mathrm{g})$ was injected intravitreally into one eye and approximately $1 \mu \mathrm{l}$ PBS containing anti-human Fc $(5 \mu \mathrm{g})$ was injected intravitreally into the contralateral eye of the same animal.

Cell culture and siRNA transfection. hDLECs were isolated and cultured as previously described (57) with the approval of the Uni- 
A

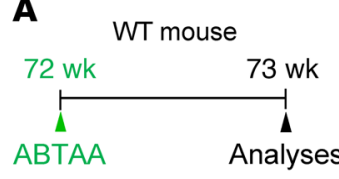

B

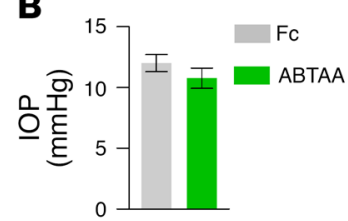

C Prox1 CD144
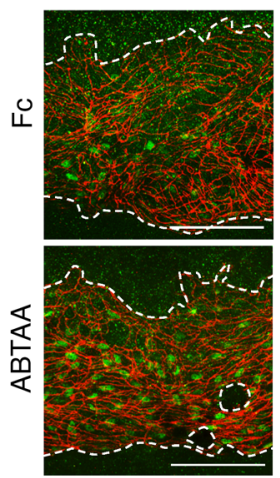

CD144
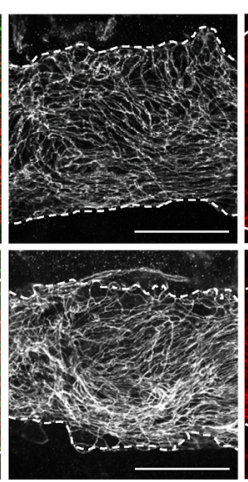

Tie2
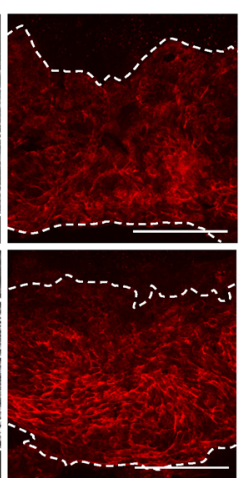

pTie2
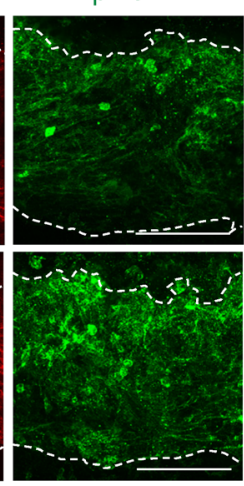

Erg
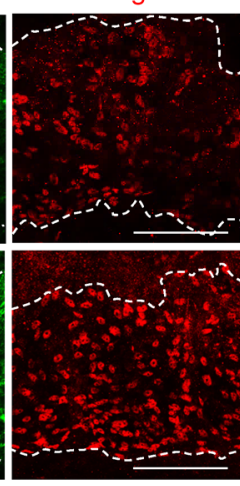

Ki-67
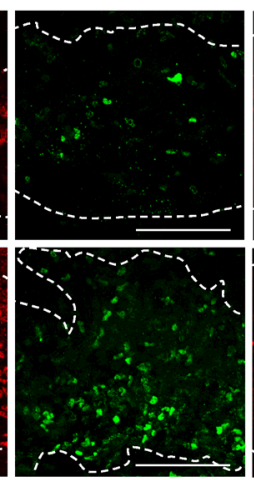

Klf4
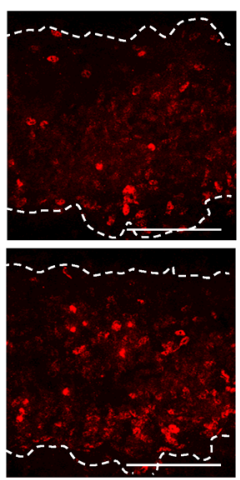
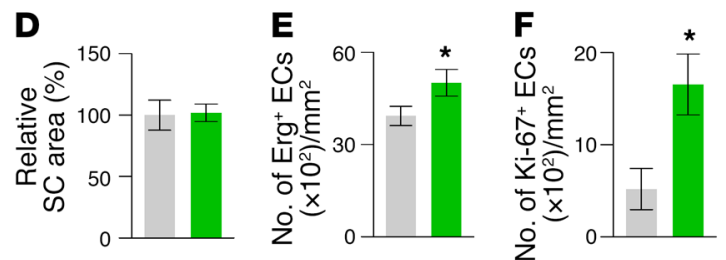

G
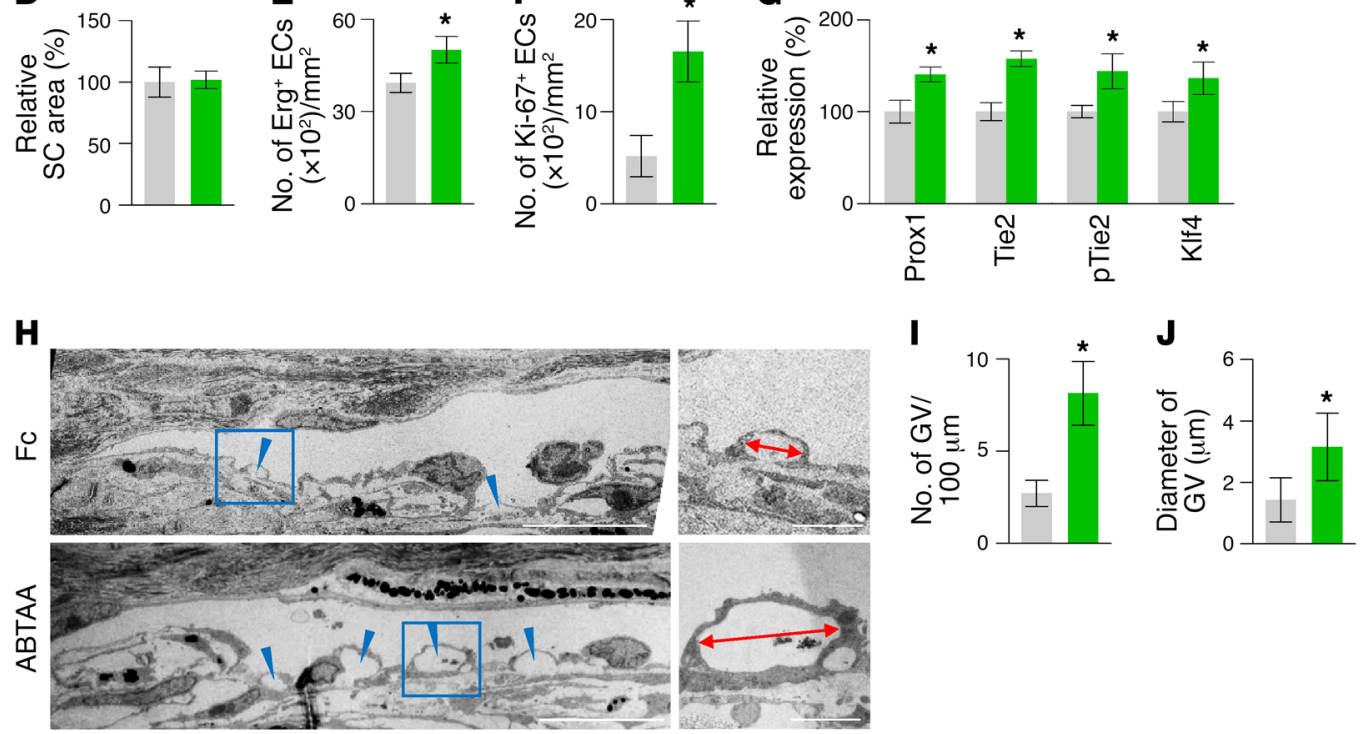

Figure 15. Tie2 activation rejuvenates SC in aged mice. (A) Diagram depicting the experiment schedule in 72-week-old WT mice for intraocular administration of ABTAA ( $\sim \mu \mathrm{g}$, left eye) and Fc ( 5 $\mu \mathrm{g}$, right eye) and analyses 1 week later. (B-G) Images and comparisons of IOP, relative area, number of Erg and Ki-67+ ECs, and intensities of Prox1, Tie2, p-Tie2, and KIf4 immunostaining in CD144+ SC. Dashed lines demarcate SC. Scale bars: 100 mm. Area and expression of each molecule in Fc-treated SC are normalized to $100 \%$, and their relative levels of ABTAA-treated SC are presented. $n=4$ for each group. ${ }^{*} P<0.05$ versus Fc by Mann-Whitney $U$ test. (H-J) EM images and comparisons of the number and diameter of $C V s$ (blue arrowheads) in the inner SC wall. Blue-lined box is magnified in right panel. Red double arrows indicate diameters of GV. Scale bars: $10 \mu \mathrm{m}$ (left panels); $2 \mu \mathrm{m}$ (right panels). $n=4$ for each group. ${ }^{*} P<0.05$ versus Fc by Mann-Whitney $U$ test.

versity of Southern California Institutional Review Board (to Y.K. Hong). In brief, the cells were confirmed to be mycoplasma negative (MycoAlert Detection Kit, Lonza), cultured in endothelial growth medium (EGM2-MV, Lonza), and incubated in a humidified atmosphere of $5 \% \mathrm{CO}_{2}$ at $37^{\circ} \mathrm{C}$. The passages of hDLECs used for all in vitro experiments were 4 to 7 . For knockdown experiments, hDLECs were transfected with siRNA as previously described (58). siRNAs targeting human PROX1, TIE2, or a scrambled control were used. Sequence information for siRNAs is shown in Supplemental Table 1. Transfections of siRNA duplexes into the hDLECs were performed using Lipofectamine RNAiMAX (Invitrogen) at a final concentration of $40 \mathrm{nM}$ for 24 to 48 hours according to the manufacturer's protocol. Then siRNA was washed out and cells were harvested 72 hours after the start of transfection.

RNA extraction and quantitative real-time RT-PCR. Total RNA of lung, limbus, or central portion of the cornea was extracted using TRIzol Reagent (Invitrogen) according to the manufacturer's instructions. For extraction of total RNA from the cultured hDLECs, RiboEx (GeneAll) was used. RNA $(2 \mu \mathrm{g})$ was reverse transcribed into cDNA using GoScript Reverse Transcription Kit (Promega). Then quantitative real-time PCR 
A

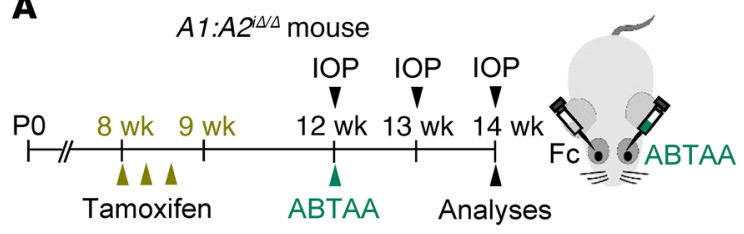

C

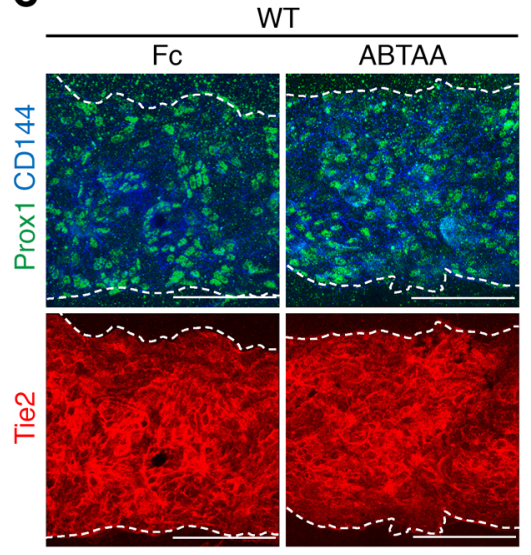

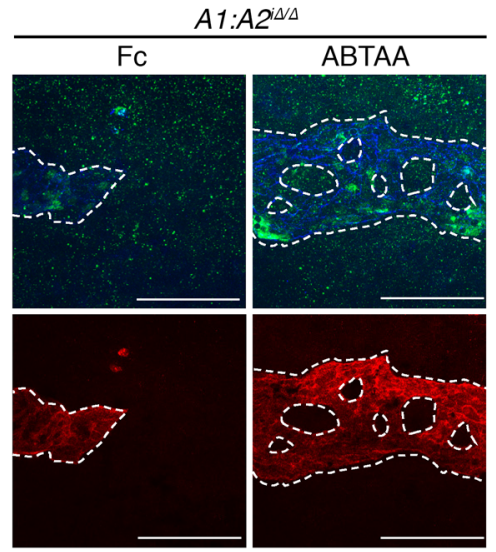
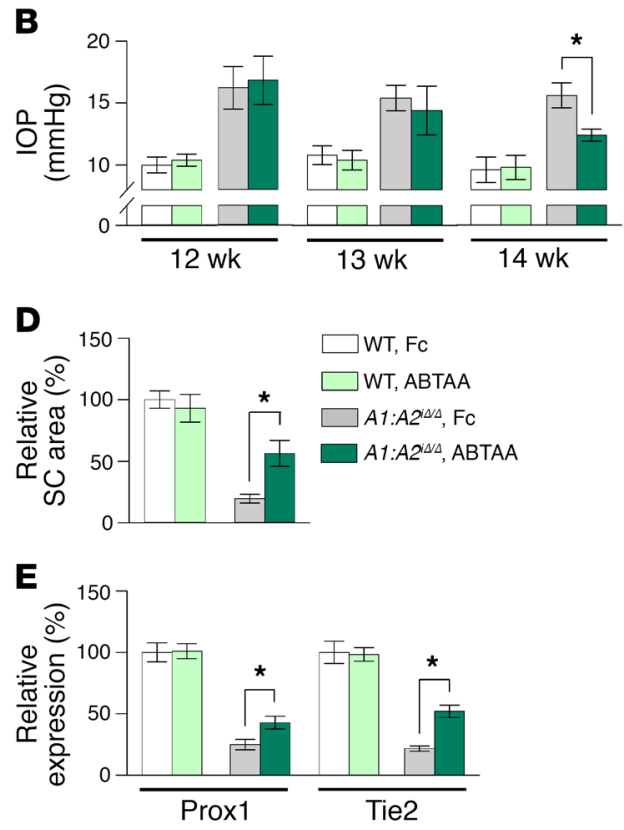

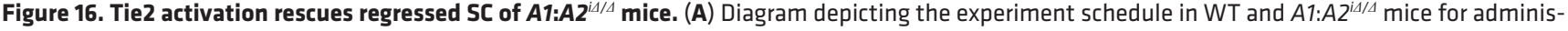
tration of tamoxifen, intraocular ABTAA ( $5 \mu \mathrm{g}$, left eye), and Fc ( $5 \mu \mathrm{g}$, right eye), periodic measurements of IOP, and analyses of their SCs. (B-E) Images and comparisons of IOP, relative area, and intensities of Prox1 and Tie2 immunostaining in CD144+ SC. Dashed lines demarcate SC. Scale bars: $100 \mu$ m. SC area and expression of each molecule in WT mice treated with Fc are normalized to $100 \%$, and relative levels of other groups are presented. $n=5$ for each group. ${ }^{*} P<0.05$ by Kruskal-Wallis test followed by Tukey's HSD test with ranks.

was performed using FastStart SYBR Green Master Mix (Roche) and S1000 Thermocycler (Bio-Rad) with the indicated primers (Supplemental Table 2). The real-time PCR data were analyzed with Bio-Rad CFX Manager Software (Bio-Rad). GAPDH was used as a reference gene, and the results were presented as relative expression to control.

RNA-seq. The cultured hDLECs were incubated with siPROX1, siTIE2, or siControl according to the aforementioned protocol, and RNA-seq of the incubated cells was performed as previously described (59). In brief, $2 \mu \mathrm{g}$ total RNA was isolated and incubated with magnetic beads coated with oligo-dT. Other RNAs except mRNA were removed by washing solution. Library production was started by random hybridization of starter/stopper heterodimers to the poly(A) RNA still connected to the magnetic beads. Illumina-compatible linker sequences were contained in the starter/stopper heterodimers. By a single-tube reverse transcription and ligation reaction, the starter was extended to the next hybridized heterodimer, where the newly synthesized cDNA insert was linked to the stopper. Second-strand synthesis was carried out to liberate the library from the beads, and then the library was amplified. We barcoded the amplified library. High-throughput sequencing was conducted as paired-end 100 sequencing using HiSeq 2000 (Illumina). Mapping of RNA-seq reads was performed using the TopHat software tool to acquire the alignment file, which was used to bring together transcripts, assess their exuberances, and identify DEGs or isoforms using cufflinks. The Ingenuity Pathway Analysis tool (QIAGEN) was used to interpret data in the context of canonical pathways, biological processes, and networks. Significance of the canonical pathways and biological function and networks were tested by the Benjamini-Hochberg procedure, which adjusts the $P$ value to correct for multiple comparisons, and their activation or inhibition was determined with reference to activation $\mathrm{z}$-scores. The activation scores and gene sets are indicated in Supplemental Tables 3 and 4. RNA-seq gene expression heatmap was generated using Cluster and TreeView from the Eisen laboratory (60). We used Multiple Experiment Viewer (MeV) from The Institute of Genomic Research (TIGR) to plot gene expression heatmap of genes encoding biological functional terms (http://mev.tm4.org/\#/welcome).

IF staining of cultured hDLECs. Indicated hDLECs plated in an 8-well chamber (Lab-Tek, Thermo Fisher Scientific) were fixed with 1\% PFA in PBS at RT for 10 minutes, permeabilized with PBST $(0.5 \%$ Triton X-100 in PBS) at RT for 5 minutes, blocked with 5\% donkey serum in PBST (0.1\% Triton X-100 in PBS) at RT for 30 minutes, and incubated with the following primary antibodies at $4^{\circ} \mathrm{C}$ overnight: anti-VE-cadherin (CD144) (mouse monoclonal, clone BV6, catalog MABT134, Millipore) and anti-PROX1 (goat polyclonal, catalog AF2727, R\&D systems). The cells were then incubated with secondary antibodies (Jackson ImmunoResearch) in the dark at RT for 1 hour and mounted with DAPI-containing mounting medium (DAKO). Images were taken with a confocal laser scanning microscope (LSM 780, Carl Zeiss).

Immunoblot analysis. To evaluate the changes in PROX1 expression in hDLECs, cells were incubated with control siRNA or siTIE2 for 48 hours and harvested at 72 hours after the start of transfection. For immunoblot detection of TIE2 downstream signaling proteins, ERK, p-ERK (T202/Y204, p-ERK), AKT, and p-AKT (S473, p-AKT) hDLECs were incubated with a premixed solution containing $2 \mu \mathrm{g} / \mathrm{ml}$ human ANGPT2 (R\&D systems) and $10 \mu \mathrm{g} / \mathrm{ml}$ ABTAA for 30 minutes without serum starvation as previously described (41). To examine changes in PROX1 expression by ABTAA treatment, hDLECs were treated with premix solutions containing IFN- $\gamma$ (Peprotech) -as an inhibitor of PROX1- and $2 \mu \mathrm{g} /$ 


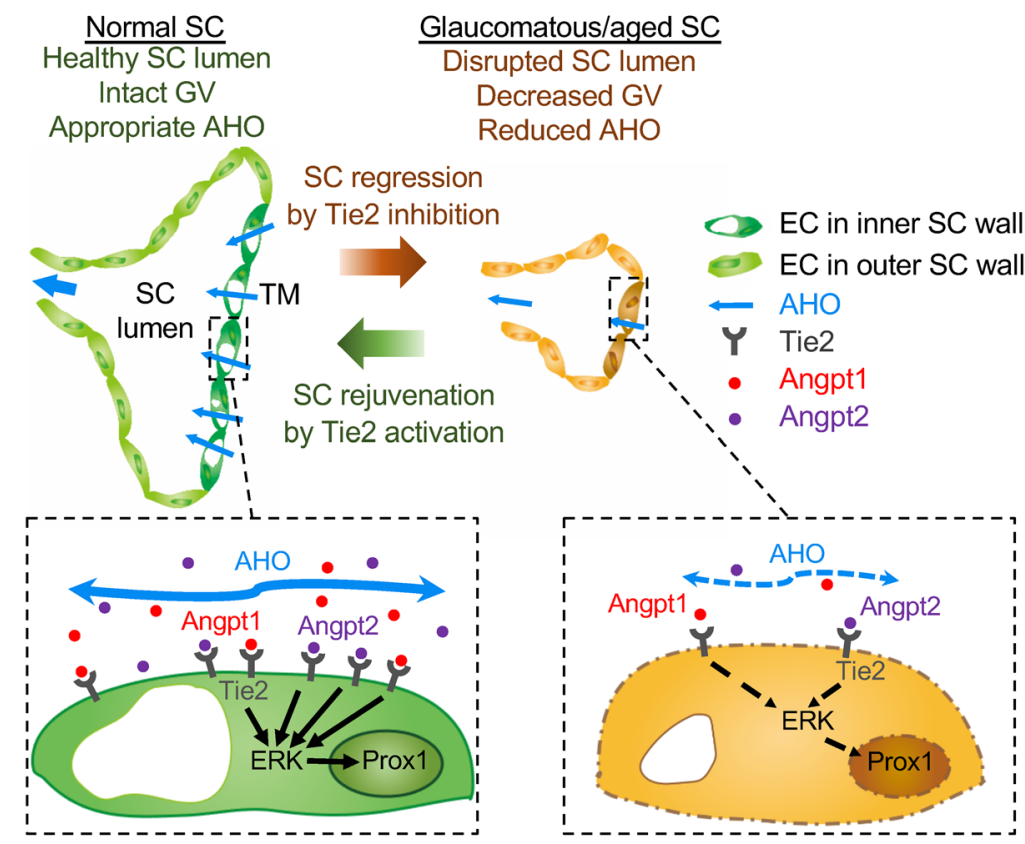

Figure 17. Schematic diagrams depicting how impairment of Angpt-Tie2 signaling disintegrates the maintenance of SC integrity, leading to glaucomagenesis. In normal adult SC, controlled Angpt-Tie2-ERK-Prox1 signaling cascade maintains appropriate $\mathrm{AHO}$ and intact GV. On the other hand, in glaucomatous or aged SC, inhibition of Angpt-Tie2 signaling attenuates ERK-Prox1 signaling and subsequently disrupts SC lumen, which consequently leads to decreased AHO. This, in turn, reduces transport of Angpt1 and Angpt2 through $\mathrm{AH}$, resulting in further inhibition of the Angpt-Tie2 system in a vicious cycle manner. These exacerbating processes eventually trigger further disintegration of SC, inducing elevated IOP and accelerating POAG progression. Tie2 activation rejuvenates SC through upregulation of ERK-Prox1 signaling, which promotes $\mathrm{AHO}$ while decreasing IOP and eventually prevents POAG progression. $\mathrm{ml}$ human ANGPT2 and $10 \mu \mathrm{g} / \mathrm{ml}$ ABTAA with or without either $50 \mu \mathrm{M}$ of ERK inhibitor U0126 (Sigma-Aldrich) and $50 \mu \mathrm{M}$ of PI3 kinase inhibitor LY294002 (Sigma-Aldrich) in 0.5\% DMSOcontaining media for 12 hours. hDLECs cultured in 0.5\% DMSO media without aforementioned agents were used as control. The cells were rinsed once with cold PBS and lysed in cold cOmplete Lysis-M Buffer (Roche) containing protease and phosphatase inhibitors (Roche). The lysates were separated by SDS-PAGE gel and transferred to nitrocellulose membranes. After blocking with $4 \%$ skim milk, the membranes were incubated with the following primary antibodies at $4^{\circ} \mathrm{C}$ overnight: anti-ERK, anti-p-ERK, anti-AKT, and anti-p-AKT (rabbit polyclonal, catalog 9102, cata$\log 9101$, catalog 9272, and catalog 9271, all from Cell Signaling Technology); anti- $\beta$-actin (rabbit monoclonal, clone C4, catalog SC-47778, Santa Cruz Biotechnology Inc.); and anti-PROX1 (goat polyclonal, catalog AF2727, R\&D systems). Membranes were then incubated with HRP-conjugated secondary antibodies (Santa Cruz Biotechnology Inc.) at RT for 1 hour. Chemiluminescent signals were developed with HRP substrate (Millipore). Resultant bands from 5 independent experiments were detected with a luminescent image analyzer (LAS-1000 mini, Fujifilm), and band intensities of indicated proteins normalized to $\beta$-actin in each lane were compared using Image J software (NIH).

Data availability. All original microarray data were deposited in the NCBI's GEO (GSE83299).

Statistics. No statistical method was used to predetermine the sample size. The investigators were blind to the genotypes of animals during experiments. Animals or samples were not randomized during experiments and were not excluded from analyses. All parameters of genetically modified mice were compared with those of littermate controls. Male and female mice were not distinguished in neonatal mice, while only male mice were used in adult experiments. Values were presented as mean \pm SD. Statistical significance was determined by the 2-sided Mann-Whitney $U$ test between 2 groups or the Kruskal-Wallis test followed by Tukey's honest significant differ- ence (HSD) test with ranks for multiple-group comparison. Statistical analysis was performed with PASW statistics 18 (SPSS). Statistical significance was set at $P<0.05$.

Study approval. Animal care and experimental procedures were performed with the approval of the Institutional Animal Care and Use Committee (no. KA2015-15) of KAIST, and mice were handled in accordance with the Association for Research in Vision and Ophthalmology Statement for the Use of Animals in Ophthalmic and Vision Research (http://www.arvo.org/About_ARVO/Policies/Statement_for_the_Use_ of_Animals_in_Ophthalmic_and_Visual_Research/).

\section{Author contributions}

JK, Dae-Young Park, HB, Do Young Park, DK, CKL, SS, and GYK designed and performed the experiments and analyzed the data. TYC and DHL provided the human samples and critical comments on this study. YK, YKH, YH, HGA, and GO provided the mice and critical comments on this study. JK, Dae-Young Park, and GYK generated the figures and wrote and edited the manuscript. GYK directed and supervised the project.

\section{Acknowledgments}

We thank Intae Park for proofreading of the manuscript, Yoshikazu Nakaoka (Osaka University) for providing Angpt1 $1^{f l / f l}$ mice, Sean J. Morrison (University of Texas Southwestern) for providing Angpt1-GFP mice, Nicholas Gale (Regeneron Pharmaceuticals) for providing Angpt2-lacZ mice, and Masanori Hirashima (Kobe University) for providing Vegfr $2^{f / f l}$ mice. This study was supported by the Institute of Basic Science (IBSR025-D1-2015 to GYK) and funded by the Ministry of Science and ICT, Republic of Korea.

Address correspondence to: Gou Young Koh, Center for Vascular Research, IBS; Graduate School of Medical Science and Engineering, KAIST, 291 Daehak-ro, Daejeon 34141, Republic of Korea. Phone: 82.42.350.2638; Email: gykoh@kaist.ac.kr. 
1. Tham YC, Li X, Wong TY, Quigley HA, Aung T, Cheng CY. Global prevalence of glaucoma and projections of glaucoma burden through 2040: a systematic review and meta-analysis. Ophthalmology. 2014;121(11):2081-2090.

2. Weinreb RN, Aung T, Medeiros FA. The pathophysiology and treatment of glaucoma: a review. JAMA. 2014;311(18):1901-1911.

3. Goel M, Picciani RG, Lee RK, Bhattacharya SK. Aqueous humor dynamics: a review. Open Ophthalmol J. 2010;4:52-59.

4. Gabelt BT, Kaufman PL. Changes in aqueous humor dynamics with age and glaucoma. Prog Retin Eye Res. 2005;24(5):612-637.

5. Janssen SF, et al. The vast complexity of primary open angle glaucoma: disease genes, risks, molecular mechanisms and pathobiology. Prog Retin Eye Res. 2013;37:31-67.

6. Ramos RF, Hoying JB, Witte MH, Daniel Stamer W. Schlemm's canal endothelia, lymphatic, or blood vasculature? J Glaucoma. 2007;16(4):391-405.

7. Truong TN, Li H, Hong YK, Chen L. Novel characterization and live imaging of Schlemm's canal expressing Prox-1. PLoS ONE. 2014;9(5):e98245.

8. Park DY, et al. Lymphatic regulator PROX1 determines Schlemm's canal integrity and identity. J Clin Invest. 2014;124(9):3960-3974.

9. Aspelund A, et al. The Schlemm's canal is a VEGF-C/VEGFR-3-responsive lymphatic-like vessel. J Clin Invest. 2014;124(9):3975-3986.

10. Kizhatil K, Ryan M, Marchant JK, Henrich S, John SW. Schlemm's canal is a unique vessel with a combination of blood vascular and lymphatic phenotypes that forms by a novel developmental process. PLoS Biol. 2014;12(7):e1001912.

11. Tammela T, Alitalo K. Lymphangiogenesis: molecular mechanisms and future promise. Cell. 2010;140(4):460-476.

12. Kwon YH, Fingert JH, Kuehn MH, Alward WL. Primary open-angle glaucoma. $N$ Engl JMed. 2009;360(11):1113-1124.

13. Streilein JW. Ocular immune privilege: therapeutic opportunities from an experiment of nature. Nat Rev Immunol. 2003;3(11):879-889.

14. Gong H, Tripathi RC, Tripathi BJ. Morphology of the aqueous outflow pathway. Microsc Res Tech. 1996;33(4):336-367.

15. Leak LV, Burke JF. Ultrastructural studies on the lymphatic anchoring filaments. JCell Biol. 1968;36(1):129-149.

16. Wigle JT, Oliver G. Prox1 function is required for the development of the murine lymphatic system. Cell. 1999;98(6):769-778.

17. Wigle JT, et al. An essential role for Prox 1 in the induction of the lymphatic endothelial cell phenotype. EMBO J. 2002;21(7):1505-1513.

18. Augustin HG, Koh GY, Thurston G, Alitalo K. Control of vascular morphogenesis and homeostasis through the angiopoietin-Tie system. Nat Rev Mol Cell Biol. 2009;10(3):165-177.

19. Koh GY. Orchestral actions of angiopoietin-1 in vascular regeneration. Trends $\mathrm{Mol} \mathrm{Med}$. 2013;19(1):31-39.

20. Thurston G, Daly C. The complex role of angiopoietin-2 in the angiopoietin-tie signaling pathway. Cold Spring Harb Perspect Med. 2012;2(9):a006550.

21. Jeansson M, et al. Angiopoietin-1 is essen- tial in mouse vasculature during development and in response to injury. J Clin Invest. 2011;121(6):2278-2289.

22. Gale NW, et al. Angiopoietin-2 is required for postnatal angiogenesis and lymphatic patterning, and only the latter role is rescued by Angiopoietin-1. Dev Cell. 2002;3(3):411-423.

23. Dellinger $\mathrm{M}$, et al. Defective remodeling and maturation of the lymphatic vasculature in Angiopoietin-2 deficient mice. Dev Biol. 2008;319(2):309-320.

24. Zheng W, et al. Angiopoietin 2 regulates the transformation and integrity of lymphatic endothelial cell junctions. Genes Dev. 2014;28(14):1592-1603.

25. Chu M, et al. Angiopoietin receptor Tie2 is required for vein specification and maintenance via regulating COUP-TFII. Elife. 2016;5:e21032.

26. Thomson BR, et al. A lymphatic defect causes ocular hypertension and glaucoma in mice. JClin Invest. 2014;124(10):4320-4324.

27. Souma T, et al. Angiopoietin receptor TEK mutations underlie primary congenital glaucoma with variable expressivity. JClin Invest. 2016;126(7):2575-2587.

28. Sabine A, et al. Mechanotransduction, PROX1, and FOXC2 cooperate to control connexin 37 and calcineurin during lymphatic-valve formation. Dev Cell. 2012;22(2):430-445.

29. Atkins GB, Jain MK. Role of Krüppel-like transcription factors in endothelial biology. Circ Res. 2007;100(12):1686-1695.

30. Choi I, et al. Visualization of lymphatic vessels by Prox1-promoter directed GFP reporter in a bacterial artificial chromosome-based transgenic mouse. Blood. 2011;117(1):362-365.

31. Overby DR, Stamer WD, Johnson M. The changing paradigm of outflow resistance generation: towards synergistic models of the JCT and inner wall endothelium. Exp Eye Res. 2009;88(4):656-670.

32. Brilakis HS, Johnson DH. Giant vacuole survival time and implications for aqueous humor outflow. J Glaucoma. 2001;10(4):277-283.

33. Zhou BO, Ding L, Morrison SJ. Hematopoietic stem and progenitor cells regulate the regeneration of their niche by secreting Angiopoietin-1. Elife. 2015;4:e05521.

34. Okabe K, et al. Neurons limit angiogenesis by titrating VEGF in retina. Cell. 2014;159(3):584-596.

35. Savant $\mathrm{S}$, et al. The orphan receptor tie1 controls angiogenesis and vascular remodeling by differentially regulating tie 2 in tip and stalk cells. Cell Rep. 2015;12(11):1761-1773.

36. Arita Y, et al. Myocardium-derived angiopoietin-1 is essential for coronary vein formation in the developing heart. Nat Commun. 2014;5:4552.

37. Lee J, et al. Angiopoietin-1 guides directional angiogenesis through integrin $\alpha \mathrm{v} \beta 5$ signaling for recovery of ischemic retinopathy. Sci Transl Med. 2013;5(203):203ra127.

38. Shen B, et al. Genetic dissection of tie pathway in mouse lymphatic maturation and valve development. Arterioscler Thromb Vasc Biol. 2014;34(6):1221-1230.

39. Harvey NL, et al. Lymphatic vascular defects promoted by Prox1 haploinsufficiency cause adult-onset obesity. Nat Genet. 2005;37(10):1072-1081
40. Davis ME, Cai H, Drummond GR, Harrison DG. Shear stress regulates endothelial nitric oxide synthase expression through c-Src by divergent signaling pathways. Circ Res. 2001;89(11):1073-1080.

41. Han S, et al. Amelioration of sepsis by TIE2 activation-induced vascular protection. Sci Transl Med. 2016;8(335):335ra55.

42. Caprioli J. Glaucoma: a disease of early cellular senescence. Invest Ophthalmol Vis Sci. 2013;54(14):ORSF60-ORSF67.

43. Aihara M, Lindsey JD, Weinreb RN. Aqueous humor dynamics in mice. Invest Ophthalmol Vis Sci. 2003;44(12):5168-5173.

44. Zhang Y, Davidson BR, Stamer WD, Barton JK, Marmorstein LY, Marmorstein AD. Enhanced inflow and outflow rates despite lower IOP in bestrophin-2-deficient mice. Invest Ophthalmol Vis Sci. 2009;50(2):765-770.

45. Crowston JG, Aihara M, Lindsey JD, Weinreb RN. Effect of latanoprost on outflow facility in the mouse. Invest Ophthalmol Vis Sci. 2004;45(7):2240-2245.

46. Millar JC, Phan TN, Pang IH, Clark AF. Strain and Age Effects on Aqueous Humor Dynamics in the Mouse. Invest Ophthalmol Vis Sci. 2015;56(10):5764-5776.

47. Johnson M, McLaren JW, Overby DR. Unconventional aqueous humor outflow: A review. Exp Eye Res. 2017;158:94-111.

48. Xin C, Johnstone M, Wang N, Wang RK. OCT study of mechanical properties associated with trabecular meshwork and collector channel motion in human eyes. PLoS One. 2016;11(9):e0162048.

49. Allingham RR, de Kater AW, Ethier CR. Schlemm's canal and primary open angle glaucoma: correlation between Schlemm's canal dimensions and outflow facility. Exp Eye Res. 1996;62(1):101-109.

50. Stamer WD, et al. Biomechanics of Schlemm's canal endothelium and intraocular pressure reduction. Prog Retin Eye Res. 2015;44:86-98.

51. Yan X, Li M, Chen Z, Zhu Y, Song Y, Zhang H. Schlemm's canal and trabecular meshwork in eyes with primary open angle glaucoma: a comparative study using high-frequency ultrasound biomicroscopy. PLOS ONE. 2016;11(1):e0145824.

52. Wang F, et al. Comparison of Schlemm's canal's biological parameters in primary open-angle glaucoma and normal human eyes with swept source optical. J Biomed Opt. 2012;17(11):116008.

53. Abu-Hassan DW, Acott TS, Kelley MJ. The Trabecular Meshwork: A Basic Review of Form and Function. J Ocul Biol. 2014;2(1):9.

54. Liton PB, Challa P, Stinnett S, Luna C, Epstein DL, Gonzalez P. Cellular senescence in the glaucomatous outflow pathway. Exp Gerontol. 2005;40(8-9):745-748.

55. Hooper AT, et al. Engraftment and reconstitution of hematopoiesis is dependent on VEGFR2mediated regeneration of sinusoidal endothelial cells. Cell Stem Cell. 2009;4(3):263-274.

56. Reitsamer HA, Kiel JW, Harrison JM, Ransom NL, McKinnon SJ. Tonopen measurement of intraocular pressure in mice. Exp Eye Res. 2004;78(4):799-804.

57. Choi I, et al. 9-cis retinoic acid promotes lymphangiogenesis and enhances lymphatic vessel 
regeneration: therapeutic implications of 9-cis retinoic acid for secondary lymphedema. Circulation. 2012;125(7):872-882.

58. Kim C, et al. Vascular RhoJ is an effective and selective target for tumor angiogenesis and vascular disruption. Cancer Cell. 2014;25(1):102-117.
59. Park JS, et al. Normalization of tumor vessels by Tie 2 activation and Ang2 inhibition enhances drug delivery and produces a favorable tumor microenvironment. Cancer Cell. 2016;30(6):953-967.

60. Eisen MB, Spellman PT, Brown PO, Botstein D.
Cluster analysis and display of genome-wide expression patterns. Proc Natl Acad Sci U S A. 1998;95(25):14863-14868.

61. Kataru RP, et al. T lymphocytes negatively regulate lymph node lymphatic vessel formation. Immunity. 2011;34(1):96-107. 